\title{
JUNCTION OF NONCOMPOSITE POLYHEDRA
}

\author{
A. V. TIMOFEENKO
}

To my son's coming of age

\begin{abstract}
All 3-dimensional convex regular-hedra are found, i.e., the convex polyhedra having positive curvature of each vertex and such that every face is either a regular polygon or is composed of two regular polygons. The algorithm for constructing such solids is based on calculation of the corresponding symmetry groups and gives a listing of all possible adjoins along entire faces of convex regular-hedra that cannot be cut by any plane into smaller regular-hedra.
\end{abstract}

\section{INTRODUCTION}

By a regular-hedron we mean a polyhedron such that the sum of the plane angles at each vertex is less than $2 \pi$, and every face is either a convex regular polygon or is composed of two convex regular polygons. In the present paper we find all 3-dimensional convex regular-hedra up to similarity.

As far back as in 1946, being in Tashkent, L. N. Esaulova sent a letter to the geometry department of Leningrad University; in that letter she proved that, besides two infinite series of equiangular-semiregular prisms $\Pi_{3}, \Pi_{5}, \Pi_{6}, \ldots$ (with regular bases and square lateral faces) and antiprisms $A_{4}, A_{5}, \ldots$ (with a belt of faces that are regular triangles), there exist only finitely many convex regular-hedra without conditional edges (a conditional edge is a common edge of two faces lying in one and the same plane). Esaulova listed the possible types of vertices of convex regular-hedra without conditional edges, and presented schemes of several such polyhedra.

In 1961, this letter reached the hands of V. A. Zalgaller. He introduced the term regular-faced polyhedra for the same polyhedra that are named "convex regular-hedra without conditional edges" in the present paper, and he distinguished between simple and composite regular-faced polyhedra. We carry these notions, which are basic for our investigation, from regular-faced polyhedra over to regular-hedra. If there exists a plane that splits a convex regular-hedron into two regular-hedra, then the initial regular-hedron is said to be composite; otherwise it is noncomposite [7, or simple 2. A description of the noncomposite regular-faced polyhedra had been obtained by 1966 as a result of efforts of many persons (see 2, and also lists of errata in 1, 3, 4 and the translation 12]). It turned out that, besides the prisms and antiprisms mentioned above, there exist only 28 noncomposite regular-faced polyhedra; they were denoted by $M_{1}, M_{2}, \ldots, M_{28}$ in the order of their arising in the classification proof. In 9] these polyhedra were called the Zalgaller solids.

2000 Mathematics Subject Classification. Primary 52B10.

Key words and phrases. Regular-hedra, concomposite polyhedra, symmetry groups, superfundamental faces.

Supported by grant 09-09-1/NSh from the V. P. Astaf'ev Krasnoyarsk State Pedagogical University, and also by grants 09-01-00395-a and 09-01-00717-a from RFBR. 
Independently of Esaulova, the same question was posed by N. Johnson, who advanced much farther. Apparently, he argued heuristically; besides the regular and equiangularsemisimple polyhedra, he found 92 regular-faced polyhedra, now called the Johnson solids. In his paper [11] he gave a name to each of them and conjectured that this list of 92 polyhedra is complete. The completeness of that list was stated in [2] on page 18 as a theorem; however, it was supplied with only a brief indication to a method of proof, namely, the listing of all possible adjoins of noncomposite polyhedra without conditional edges along entire faces.

In the papers [3, 4, which continued the study in [2, all noncomposite polyhedra with a face containing a conditional edge were found. These regular-hedra were denoted by $Q_{1}, Q_{2}, \ldots, Q_{6}$. Any conditional edge of every such solid connects triangles. We call the polyhedron $Q_{6}$ (Figure [36) the Pryakhin solid, and the polyhedra $Q_{1}, Q_{2}, Q_{3}, Q_{4}$, $Q_{5}$ the Ivanov solids.

Thus, in the beginning of the 1970s, we had a list of all noncomposite solids

$$
\Pi_{3}, \Pi_{4}, \ldots ; A_{4}, A_{5}, \ldots ; M_{1}, M_{2}, \ldots, M_{28} ; Q_{1}, Q_{2}, \ldots, Q_{6} .
$$

However, for a person who wants to verify that no other noncomposite solids exist, it would be necessary to read more than 200 pages and to check the validity of the calculations. But throughout the last decades, such difficulties of classification proofs have been avoided with the help of computer algebra systems. Indeed, an application of such systems allows one to verify the underlying algorithm, instead of arguments and calculations determined by this algorithm, provided, of course, that we trust the computer system itself.

The algorithm for constructing all composite solids, applied in the present paper, works without a computer, though it involves a description of algebraic models of polyhedra and their symmetry groups, obtained via the computer algebra systems GAP 1 and Maple.

The construction of all convex regular-hedra via a listing of adjoins of noncomposite polyhedra along entire faces was announced by the author [5, 8] and by the author and Gurin [9, 10] (Gurin worked together with V. A. Zalgaller). However, the listing was accomplished by them in parallel. As a result, two proofs appeared for the theorem that describes the convex regular-hedra, in the paper [1] and in the present paper. The authors of [1] explored convex regular-hedra under the weaker condition that more than one conditional edge may belong to some of the faces; this led to the arising of the following two composite solids: $\Pi_{3}+\Pi_{4}+\Pi_{3}^{\prime}$ and $\Pi_{3}+\Pi_{5}+\Pi_{3}$. On the other hand, six composite solids were only found with the help of the author's composition algorithm, which was indicated in [1].

It turned out that there are 149 composite polyhedra, and among them 75 are Johnson solids. Adding to the latter 17 noncomposite Johnson solids, namely, the solids $M_{k}$ with $k=2, \ldots, 9,13,14,20,21, \ldots, 25,28$, we get the proof of Johnson's conjecture.

\section{§1. Definitions, notation, Known facts}

Definition 1. A polygon is part of the plane bounded by a broken line. An equilateral and equiangled polygon is regular.

Definition 2. A polyhedron in the 3-dimensional Euclidean space is a finite collection of polygons with the following properties: 1) every side of any polygon is simultaneously a side of precisely one other polygon (this latter is said to be adjacent to the former along that side); 2) starting with any polygon, we can reach any other of them by passing consequently from a polygon to another polygon adjacent to it. These polygons are

\footnotetext{
${ }^{1}$ http://www-gap.dcs.st-and.ac.uk/ gap.
} 
called faces, and their sides are edges. A polyhedron is convex if it lies entirely in one half-space determined by the plane passing through any of its faces.

In this paper, a convex polyhedron is regarded as a solid formed by all points of its faces and also all points lying inside of the polyhedron.

Definition 3. A convex polyhedron is regular if equally many edges emanate from each vertex and all faces are regular polygons with the same number of sides.

It is well known that there are only 5 regular polyhedra, namely, 4-, 6-, 8-, 12-, and 20-faced Platonic solids.

Definition 4. By an equiangled-semiregular polyhedron we mean a convex polyhedron with the following properties: each face of it is a regular polygon, but there are faces with different numbers of sides, and all vertices are alike in the sense that any two of them can be identified by a self-matching of the polyhedron.

Besides infinite series of prisms and antiprisms, there exist only 13 equiangled-semiregular polyhedra, which are called the Archimedean solids.

It is convenient to denote each regular or equiangled-semiregular polyhedron by its vertex type, indicating in brackets the numbers of the sides of the faces incident to every vertex. For instance, $[3,3,3]$ corresponds to a tetrahedron, $[4,4, n]$ corresponds to a prism with $n$-angled base, and $[3,4,3,4]$ denotes a cuboctahedron. Of course, one-type vertices may fail to be vertices of equal polyhedral angles. As an example, we mention one-type vertices of a snub cube $[3,3,3,3,4]$ and a snub square antiprism $M_{28}$ (Figure 2).

Among the 28 Zalgaller solids, there are 9 Archimedean solids, the tetrahedron $M_{1}$, the dodecahedron $M_{15}$, and 17 Johnson solids; see Tables 11and 2, and also [7, page 137]). The following composite regular-hedra are also Archimedean solids: $M_{4}+M_{4}^{\prime}=[3,4,3,4]$, $M_{9}+M_{9}=[3,5,3,5], M_{6}+M_{14}+M_{6}=[3,4,5,4]$, and $\Pi_{8}+M_{5}+M_{5}=[3,4,4,4]$, where $\Pi_{8}=[4,4,8]$ is the prism with 8 -angled base.

Definition 5. The curvature of a vertex of a polyhedron is the difference between $2 \pi$ and the sum of the plane angles incident to that vertex. Obviously, the curvature of each vertex of a regular-hedron is positive.

Definition 6. Let $G$ be a group, and let $M$ be a set such that, for any $g \in G$ and $m \in M$, an element $g m \in M$ is defined. We recall that $G$ acts on $M$ if $\left(g_{2} g_{1}\right) m=g_{2}\left(g_{1} m\right)$ and $\varepsilon m=m$, where $g_{1}, g_{2}$ are arbitrary elements of $G, m \in M$, and $\varepsilon$ denotes the unity element of $G$. The set $G m=\{g m \mid g \in G\}$ is called the orbit of the element $m \in M$.

Definition 7. Let $k$ be a positive real number. A similarity with coefficients $k$ is a transformation of Euclidean space such that any two points at a distance of $d$ are mapped to points the distance between which is $k d$.

Definition 8. An isometry preserves the distances; thus, it is a similarity with coefficient 1.

Definition 9. If the determinant of the matrix that corresponds to an isometry is positive, then this transformation is proper, otherwise if is improper.

If a proper isometry has a fixed point, then it is a rotation.

Definition 10. The group of all rotations that identify a polyhedron $P$ with itself will be denoted by $\mathrm{Aut}^{+}(P)$, and the group of all isometries with the same property by $\operatorname{Aut}(P)$. The elements of the group Aut $(P)$ will be called the symmetries of $P$, and the elements of Aut ${ }^{+}(P)$ are the rotations of $P$. Accordingly, $\operatorname{Aut}^{+}(P)$ is the group of rotations of $P$, and $\operatorname{Aut}(P)$ is the group of symmetries of $P$. 
We recall that any finite group of isometries of 3-dimensional Euclidean space is equal to the group of symmetries of one of the following solids: the tetrahedron $[3,3,3]$, the icosahedron $[3,3,3,3,3]$, and the prisms $[4,4, n], n=3,4,5, \ldots$.

Definition 11. If the set of vertices of a polyhedron $P$ is the union of the orbits of points $T_{1}, T_{2}, \ldots, T_{k}$ under the action of a subgroup $G$ of the group $\operatorname{Aut}(P)$, and $k$ is minimal among the numbers with this property, then we say that the points listed above are fundamental vertices of the polyhedron $P$ under the action of $G$. If $G=\operatorname{Aut}(P)$, then the words "under the action of $G$ " are omitted.

Definition 12. If the set of faces of a polyhedron $P$ is equal to the union of the orbits of faces $F_{1}, F_{2}, \ldots, F_{k}$ under the action of a subgroup $G$ of the group $\operatorname{Aut}(P)$, and the number $k$ cannot be reduced, then we say that the faces listed above are fundamental faces of $P$ under the action of $G$. If $G=\operatorname{Aut}(P)$, then the words "under the action of $G$ " are omitted.

Example 1. The set of fundamental faces of a regular polyhedron consists of one polygon. For an equiangled-semiregular polyhedron, fundamental faces can be chosen incident to one vertex; the number of such faces cannot be less than two.

Example 2. The set of fundamental vertices of each Platonic or Archimedean solid consists of one point.

In the next proposition we indicate 9 fundamental vertices of the polyhedron $Q_{3}$ (see Figure 5).

Proposition 1. Reflection in a plane is the only nonidentical symmetry of the Ivanov solid $Q_{3}$, and if this plane contains the $x$ - and $z$-axes, then the points listed below are fundamental vertices of $Q_{3}$ :

$$
\begin{array}{lll}
1\left(0, \frac{1+\sqrt{5}}{4}, 0\right), & 2\left(\frac{1}{2}, \frac{1}{2}, \frac{1+\sqrt{5}}{4}\right), & 3\left(\frac{3+\sqrt{5}}{4}, 0, \frac{1}{2}\right), \\
4\left(\frac{3+\sqrt{5}}{4}, 0, \frac{-1}{2}\right), & 5\left(\frac{1}{2}, \frac{1}{2},-\frac{1+\sqrt{5}}{4}\right), \\
6\left(\frac{-1}{2}, \frac{1}{2},-\frac{1+\sqrt{5}}{4}\right), & 7\left(-\frac{\sqrt{5}}{5}, \frac{1+\sqrt{5}}{4}, \frac{7 \sqrt{5}}{20}\right), \\
8\left(-\frac{5+2 \sqrt{5}}{10}, \frac{1}{2}, \frac{2 \sqrt{5}-5}{20}\right), & 9\left(-\frac{5+7 \sqrt{5}}{20}, 0, \frac{5+2 \sqrt{5}}{10}\right) .
\end{array}
$$

For any positive integer $k$, we denote by $C_{k}$ the cyclic group of rotations about a line by angles that are multiples of $2 \pi / k$, and by $D_{2 \cdot k}$ the dihedral group generated by rotation by $2 \pi / k$ about a line and reflection in a plane containing that line. In particular, $C_{1}$ is the unit group, and $D_{2 \cdot 1}$ is the group of order 2 .

Definition 13. If $M$ is a subset in a group $G$, and each element of $G$ can be written in the form

$$
m_{1}^{\epsilon_{1}} m_{2}^{\epsilon_{2}} \ldots m_{k}^{\epsilon_{k}}, \quad m_{i} \in M, \quad \epsilon_{i}= \pm 1, \quad k=1,2, \ldots,
$$

then $M$ is a generating set for $G$.

Proposition 2. If $a$ is a root of the polynomial $26880 x^{10}+35328 x^{9}-25600 x^{8}-$ $39680 x^{7}+6112 x^{6}+13696 x^{5}+2128 x^{4}-1808 x^{3}-1119 x^{2}+494 x-47(a \approx 0.21684)$, then 
symmetries that generate the group of symmetries of the hebesphenomegacorona $M_{21}$ and its fundamental vertices can be given by the following matrices:

$$
\begin{aligned}
& \left(\begin{array}{ccc}
1 & 0 & 0 \\
0 & -1 & 0 \\
0 & 0 & 1
\end{array}\right), \quad\left(\begin{array}{ccc}
-1 & 0 & 0 \\
0 & 1 & 0 \\
0 & 0 & 1
\end{array}\right), \quad\left(\begin{array}{c}
1 / 2 \\
1 / 2 \\
\sqrt{1-a^{2}}
\end{array}\right), \\
& \left(\begin{array}{c}
1 / 2+a \\
1 / 2 \\
0
\end{array}\right), \quad\left(\begin{array}{c}
1 / 2+\sqrt{(1-2 a) /(2-2 a)} \\
\left.1-a-2 a^{2}\right) / 2 / \sqrt{1-a^{2}}
\end{array}\right), \quad\left(\begin{array}{c}
1 / 2 \\
0 \\
0 \\
-\sqrt{3-4 a^{2}} / 2
\end{array}\right), \\
& \left(\begin{array}{c}
\left(\sqrt{2} \sqrt{\left(3-4 a^{2}\right)(1-2 a)}+\sqrt{1+a}\right) / 4 /(1-a) / \sqrt{1+a} \\
(2 a-1) \sqrt{3-4 a^{2}} / 8 /(1-a)-\sqrt{2(1-2 a)} / 4 /(1-a) / \sqrt{1+a}
\end{array}\right) .
\end{aligned}
$$

In Figure 1, the fundamental vertices of the polyhedron $M_{21}$ are marked in accordance with their order in Proposition 2.

Proposition 3. The group Aut $\left(Q_{5}\right)$ is generated by two symmetries, namely, by rotation by $\pi$ about the line passing through the midpoints of the edges every end of which is incident to two triangular faces, a square face, and a rhombic face, and by reflection in the plane orthogonal to that line and passing through the midpoint of the edge common to the pentagonal and hexagonal faces (see Figure 6).

Proofs of Propositions 1, 2, 3. The deduction of all formulas can be found in [6, 7]; their validity can be checked by direct calculations. It is convenient to choose coordinate axes so that they determine planes of reflections or axes of rotations that generate the group of symmetries of the polyhedron in question. Precisely this was done in Proposition 1 and this can be done for the polyhedron $Q_{5}$ (see Proposition 3). In such cases the matrices of symmetries are found easily. Conversely, the corresponding reflections are seen from the matrices of Proposition 2

Definition 14. By an F-polyhedron we mean any convex regular-hedron such that the composition of it with every convex regular-hedron is not a convex regular-hedron.

Definition 15. Suppose we are given a polyhedron $P$. From the set of its fundamental faces, we delete all faces such that the composition along each of them of $P$ with any convex regular-hedron does not yield a convex regular-hedron. The set of remaining faces will be denoted by $S F$, and each face belonging to $S F$ is called a superfundamental face of the polyhedron $P$.

Now, an F-polyhedron is a convex regular-hedron for which the set $S F$ is empty.

In the present paper, we denote every composite polyhedron in at least two ways, in the form of a sum of noncomposite polyhedra and as one of the elements of the list (2). The first of them is a modification of the system of notation introduced in 2. The Platonic solids and the equiangled-semiregular polyhedra are also denoted by the types of their vertices, and the Johnson solids are denoted by the symbols $J_{k}, k=1,2, \ldots, 922$

Each composite polyhedron with conditional edges and with nonempty set $S F$ is provided with a name created on the basis of names introduced in [11. As a rule, this name points to the construction of the polyhedron in question and makes it possible to identify composite solids formed by equal summands.

Definition 16. Let $k$ be an integer. A composite regular-hedron is said to be $k$-composite if it can be composed along entire faces from $k$ noncomposite solids and cannot be composed from a smaller number of noncomposite solids.

\footnotetext{
${ }^{2}$ http://mathworld.wolfram. com/JohnsonSolid.html.
} 
Example 3. The icosahedron $M_{3}+M_{7}+M_{3}+M_{3}$ is a 3 -composite solid because it is not 2-composite and $[3,3,3,3,3]=A_{5}+M_{3}+M_{3}$.

Definition 17. The set of coordinate triples of the vertices of a polyhedron will be treated as the support of an algebraic system in which every $k$-angled face corresponds to a $k$-ary relation among vertices, listed in the order of their arrangement on that face. This system will be called the algebraic model of the polyhedron.

In Propositions 1, 2] 3, algebraic models are given by fundamental vertices and by symmetries that generate the corresponding groups of symmetries. For an automatic calculation of the group of symmetries of a polyhedron, it is convenient to use the algebraic model of the "skeleton" of that polyhedron. The support of such a model is the set of numbers of the vertices.

Suppose the coordinates of the vertices are given precisely, and in a form accessible to modern systems of computer algebra. Then, having fixed the length of an edge of every noncomposite polyhedron (e.g., assuming it to be of unit length), we can use a computer algebra system to solve the problem of composition of polyhedra along entire faces and to find out whether the resulting polyhedron will be convex and whether it will be isomorphic to another composite polyhedron. The collection of commands that describes the algebraic model of a polyhedron will be called the computer model of that polyhedron.

All the drawings in the present paper were made with the help of computer models created on the basis of the author's algebraic models.

When coloring faces of polyhedra, we followed the rules below.

a) The colors of the superfundamental faces and of the fundamental faces that are not superfundamental are chosen to be different: light golden and grey 3 respectively. Note that, on some drawings, the reader sees some of the fundamental faces from inside, through the net of edges located nearer.

b) For a $k$-composite polyhedron $P_{1, n_{1}}+P_{1, n_{2}}+\cdots+P_{1, n_{k}}, k \in\{2, \ldots, 6\}$, faces of each summand $P_{1, n_{i}}$ are colored with their own color corresponding to the value of $i$ : 1) red, 2) orange, 3) yellow, 4) green, 5) light blue, 6) blue. These colors are discernible in black-and-white printings. For instance, in Figure 44 the first four colors go from left to right and from bottom to top.

In the figures, some fundamental vertices are marked by numbers. Conditional edges of some polyhedra are depicted by dashed lines. "Live" images of convex regular-hedra without conditional edges, which are more visual than paper drawings, can be found in the electronic encyclopedia 4 Table 1 of the Zalgaller $F$-polyhedra will be helpful.

Remark 1. Besides rhombuses composed of two regular triangles, in the present paper we shall meet faces composed of a triangle and a square, and of a triangle and a pentagon. When calculating the number of faces, we count each "double" face of that sort as two faces, still calling it a rhombus or an irregular pentagon or hexagon, respectively.

\section{§2. Algorithm For the Synthesis of COMPosite Polyhedra}

Before describing the algorithm, we note that it will work only with 30 polyhedra in the list (1) that are indicated in Proposition 4 Then, in Subsection 2.2, we describe the step of the algorithm and introduce notation for composite polyhedra.

\footnotetext{
${ }^{3}$ When printed in black and white, the light golden color becomes darker than grey.

${ }^{4}$ http://mathworld. wolfram. com/topics/Polyhedra.html.
} 


\subsection{Noncomposite $F$-polyhedra.}

Proposition 4. Composition along faces of any noncomposite solid different from the polyhedra on the list

$$
\Pi_{3}, \ldots, \Pi_{6}, \Pi_{8}, \Pi_{10} ; A_{4}, A_{5}, A_{6}, A_{8}, A_{10} ; M_{1}, \ldots, M_{15}, M_{20}, M_{22} ; Q_{4}, Q_{6}
$$

with any convex regular-hedron is not a convex regular-hedron.

Proof. The edges and fundamental faces of the polyhedra of Proposition 4 are depicted in Figures 7 36,

We want to find out what elements of the list (1) (see the Introduction) are $F$-polyhedra; removing them from that list, we shall check Proposition 4.

The Zalgaller, Ivanov, and Pryakhin solids contain no faces with $l=7,9,11,12,13, \ldots$. sides. Therefore, every composition of noncomposite solids along $l$-angled faces is one of the polyhedra $\Pi_{l}+\Pi_{l}, \Pi_{l}+A_{l}$, or $A_{l}+A_{l}$. Since each of them has a vertex with zero curvature, it is not a regular-hedron.

Let $Q$ be a noncomposite polyhedron possessing a square face. Clearly, the composition of the solids $\Pi_{7}$ and $Q$ along square faces can be convex only if the identified square face of $Q$ makes nonobtuse angles with the faces adjacent to it along its opposite edges. This property is fulfilled only for the noncomposite polyhedra $M_{2}, \Pi_{3}, \Pi_{4}$. Joining the base of the 4-angled pyramid $M_{2}$ to a lateral face of the prism $\Pi_{7}$, we obtain a nonconvex regular-hedron. The angle between the base and a lateral face of $M_{2}$ is less than that between adjacent lateral faces of any prism, and the angle between adjacent faces of $\Pi_{7}$ is less than such an angle in a prism with a greater number of faces. Therefore, the prisms $\Pi_{l}$ with $l$ indicated above are $F$-polyhedra.

To obtain a convex regular-hedron by composition of the antiprism $A_{7}$ and a noncomposite solid along triangular faces, it is necessary that this solid have a triangular face making an acute angle with any face adjacent to it. We have such a triangular face only in the tetrahedron $M_{1}$. Its composition with $A_{6}$, and thereby, with any antiprism $A_{l}$, is not a regular-hedron.

The proof of Proposition 4 will be complete if we find all $F$-polyhedra among the Zalgaller and Ivanov solids.

The composition of every Archimedean polyhedron $M_{16}-M_{19}$ with a noncomposite polyhedron will be convex if in the latter polyhedron the face to be identified makes

TABLE 1. Zalgaller $F$-polyhedra $\mathrm{M}_{\mathrm{i}}$

\begin{tabular}{|c|l|l|}
\hline$i$ & Name & Notation \\
\hline 16 & Truncated octahedron & {$[4,6,6]$} \\
\hline 17 & $\begin{array}{l}\text { Great } \\
\text { rhombicuboctahedron }\end{array}$ & {$[4,6,8]$} \\
\hline 18 & $\begin{array}{l}\text { Great } \\
\text { rhombicosidodecahedron }\end{array}$ & {$[4,6,10]$} \\
\hline 19 & Truncated icosahedron & {$[5,6,6]$} \\
\hline 21 & Hebesphenomegacorona & $\mathrm{J}_{89}$ \\
23 & Sphenomegacorona & $\mathrm{J}_{88}$ \\
\hline 24 & Disphenocingulum & $\mathrm{J}_{90}$ \\
\hline 25 & Snub disphenoid & $\mathrm{J}_{84}$ \\
\hline 26 & Snub cube & {$[3,3,3,3,4]$} \\
\hline 27 & Snub dodecahedron & {$[3,3,3,3,5]$} \\
\hline 28 & Snub square antiprism & $\mathrm{J}_{85}$ \\
\hline
\end{tabular}


acute angles with the faces adjacent to it. The number of sides of that face is $4,5,6,8$, or 10. Therefore, it suffices to consider the following compositions:

$M_{16}=[4,6,6]$ with $M_{2}$ (Figure 19) and $M_{4}$ (Figure 21);

$M_{17}=[4,6,8]$ with $M_{2}, M_{4}$ and $M_{5}$ (Figure 222);

$M_{18}=[4,6,10]$ with $M_{2}, M_{4}, M_{6}$ (Figure 23) and $M_{9}$ (Figure 25);

$M_{19}=[5,6,6]$ with $M_{3}$ (Figure 201) and $M_{4}$.

Each of these compositions contains a vertex belonging to both parts to be joined and such that its curvature is nonpositive. Thus, the composition itself is not a regularhedron.

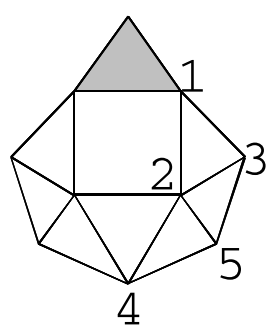

Figure $1 . M_{2}+M_{21}$.

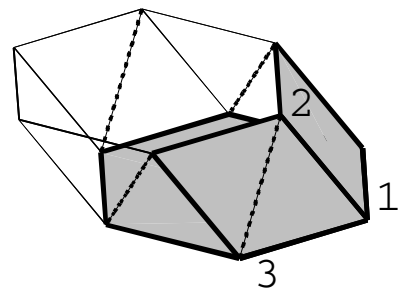

Figure $3 . Q_{1}$.

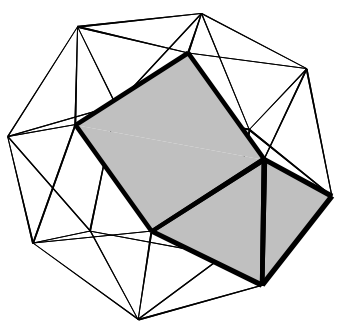

Figure 2. $M_{28}$.

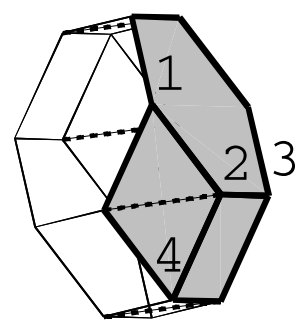

FiguRE 4. $Q_{2}$.

The hebesphenomegacorona has a single face, each vertex of which is incident to at least 5 edges. This is the square adjacent to two square faces. The algebraic model of $M_{21}$ (see Proposition 2) allows us to use the scalar triple product in order to see that the composition along the square face mentioned above of $M_{21}$ and the pyramid $M_{2}$ is a nonconvex regular-hedron. We denote it by $M_{2}+M_{21}$. Figure 1 was created on the basis of the algebraic and computer models and shows a projection of the solid $M_{2}+M_{21}$; in this projection we can see nonconvexity (a face of the pyramid is shadowed).

Each fundamental face of any of the polyhedra $M_{23}-M_{28}$ has a vertex incident to five edges. For example, for the snub square antiprism $M_{28}$ we can see this in Figure 2 , where its edges and fundamental faces are depicted. Therefore, any composition along such a face leads to arising a vertex incident to at least 6 edges, which is impossible for a regular-hedron.

In Figures 3 and 4 the digit 2 denotes the vertices of trihedral angles in the Ivanov solids $Q_{1}$ and $Q_{2}$. The plane angles at those vertices are $90^{\circ}, 120^{\circ}$, and $120^{\circ}$. They are equal to the plane angles at a vertex of the truncated octahedron $M_{16}$. For these three polyhedra, the vertices indicated above are incident to all fundamental faces. Since $M_{16}$ is an $F$-polyhedron, we conclude that so are the solids $Q_{1}$ and $Q_{2}$.

Lemma 1. The Ivanov solid $Q_{3}$ is an F-polyhedron.

Proof. By Proposition 1, the only nonidentical symmetry of $Q_{3}$ is the reflection in the plane passing through the points 3,4,9 (see Figure 5). For each of the noncomposite 

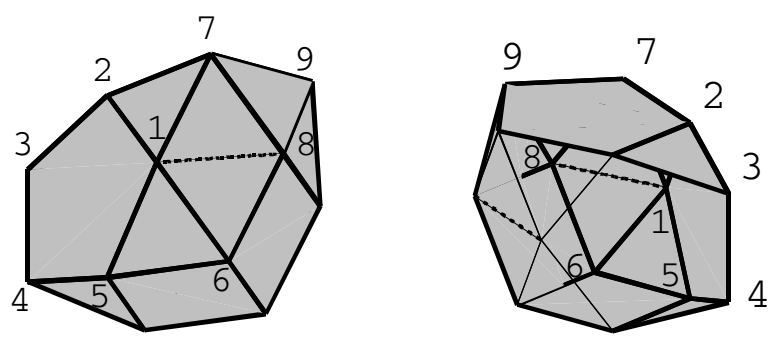

Figure 5. Edges, fundamental vertices, and 12 fundamental faces of the Ivanov solid $Q_{3}$.
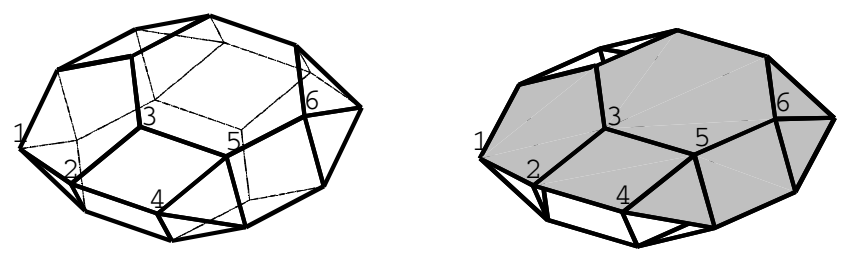

Figure 6. Edges, fundamental faces, and fundamental vertices of the solid $Q_{5}$.

polyhedra $Q_{3}-Q_{6}$, its rhombic face makes an obtuse angle with every adjacent face. Therefore, the rhombus $(1,6,8,7)$ of the solid $Q_{3}$, composed of two triangles, cannot serve as a joining face with a noncomposite polyhedron, and the vertices 6 and 8 (connected by a conditional edge) are incident to 5 edges each. Consequently, no regular-hedron can be obtained by composition with the solid $Q_{3}$ along faces containing the vertices 6 and 8 .

If $Q_{3}$ is composed with any regular-hedron along the triangle with the edge $(2,3)$, then the curvature of the vertex 2 reduces at least by $60^{\circ}$ and becomes negative. Therefore, it remains to consider the following three fundamental faces of the Ivanov solid $Q_{3}$ : the triangle with the edge $(4,5)$, the square with the edge $(5,6)$, and the pentagon with the edge $(2,7)$. Each of these faces makes obtuse angles with all faces adjacent to it. A calculation based on the algebraic model of $Q_{3}$ (see Proposition 1) shows that the compositions of $Q_{3}$ with the 3-, 4-, or 5-angled pyramids $M_{1}, M_{2}, M_{3}$ along the triangle, square, or pentagon mentioned above, respectively, are nonconvex regular-hedra. Since the other noncomposite solids have no 3-, 4-, 5-angled faces that make acute angles with all faces adjacent to them, Lemma 1 is proved.

Lemma 2. The Ivanov solid $Q_{5}$ is an $F$-polyhedron.

Proof. Proposition 3 shows that $Q_{5}$ has 7 fundamental faces (Figure6). Three of them are incident to the vertex 3 , and the plane angles at that vertex are $108^{\circ}, 120^{\circ}, 120^{\circ}$. These are the same as the plane angles at a vertex of the $F$-polyhedron $M_{19}$. Consequently, the compositions of any regular-hedron with the solid $Q_{5}$ along faces with the vertex 3 do not yield a convex regular-hedron. Taking the conditional edge $(3,4)$ into account, we see that the vertex 4 is incident to 5 edges. Thus, it remains to consider the two triangles and the square incident to the vertex 6 . The curvature of that vertex is $30^{\circ}$. If we join a regular-hedron to any face incident to that vertex, then its curvature will reduce at least by $30^{\circ}$. Therefore, the resulting composition will not be a regular-hedron.

This completes the proof of Proposition 4 
2.2. Synthesis of composite polyhedra. We agree that the first step of the algorithm for synthesizing convex regular-hedra is completed with the construction of a list of all noncomposite polyhedra, together with the set $S F$ of superfundamental faces for each of them. All noncomposite polyhedra for which the set $S F$ is nonempty are listed in Table 2 . The corresponding superfundamental faces will be indicated when we describe how our algorithm works with polyhedra, see Figures $7-36$. The next steps of the algorithm will lead to noncomposite polyhedra.

Before enriching the list of composite solids with a new polyhedron, we must check that the latter is absent from that list. A standard solution of this problem consists in the replacement of each composite polyhedron by the algebraic model of its "skeleton", followed by the answer to the question about a possible isomorphism of models. Such a model consists of a vertex set and the binary relations determined on it by the edges. At present, computer algebra systems can solve this isomorphism problem. Otherwise, without applying a computer, it is convenient to equip every polyhedron with a list of its faces, indicating the number of its 3-, 4-, 5-, 6-, 8-, and 10-angled regular faces, and also the number of faces of the form $3+3,3+4$, and $3+5$. Then we compare the corresponding lists, rather than two polyhedra themselves, turning to polyhedra only if the lists coincide. In the latter case the polyhedra in question may be different (e.g., the solids composed of two rotundas $M_{9}$ and the pyramid $M_{3}$; see Figures 39, 40, 41), and may be equal, such as $M_{3}+M_{20}$ and $M_{3}+Q_{6}$.

Suppose that at the $k$ th step we constructed all $k$-composite polyhedra, with an indication of the set $S F$ for each of them. We shall denote the $F$-polyhedra constructed at the $k$ th step by sans-serif type letters, while the other polyhedra will be written in the form of the sum of $k$ summands, and will also be denoted by the letter $P$ with two lower indices:

$$
P_{k, 1}, P_{k, 2}, \ldots, P_{k, n_{k}} .
$$

We describe the $(k+1)$ st step of the algorithm. We shall compose each polyhedron constructed at the preceding step with each polyhedron of Proposition 4, Let $P_{k}$ and $M$ be such polyhedra. If we obtain a composition that is a regular-hedron and is absent in the preceding list, we denote it by $P_{k}+M$ and add it to the list of composite solids. In some cases, we shall indicate the presence of a conditional edge by a horizontal bar: $\overline{P+M}, \overline{Q_{4}}, \overline{P_{30}}$. Composing $P_{k}$ with a polyhedron $M$ of Proposition 4 we can possibly obtain a $(k+1)$-composite solid $P_{k+1}$. Then we write

$$
P_{k+1}=P_{k}+M .
$$

The expression on the right has $k+1$ terms, the first $k$ of which form the expression $P_{k}$.

Sometimes, composing $P_{k}$ and $M$ in different ways, we get different polyhedra. Then we distinguish them by supplying the second term with a prime $P_{k}+M, P_{k}+M^{\prime}$, $P_{k}+M^{\prime \prime}, \ldots$ If the polyhedron $P_{k}$ is fixed, then each such prime can be regarded as a congruent transformation that repositions the polyhedron $M$ from one (superfundamental) face of $P_{k}$ to another face, or leaves $P_{k}$ and $M$ composed along the same face, but with other vertices identified.

After considering all compositions of $P_{k}$ and $M$, we turn to Proposition 4 to find the noncomposite polyhedron next after $M$, and add to our list all compositions of this polyhedron with $P_{k}$ that did not occur earlier. If there is no such noncomposite polyhedron, i.e., $M=Q_{6}$, then the $(k+1)$ st step of the algorithm is completed; the result is a list of all $(k+1)$-composite polyhedra. Now we delete all the $F$-polyhedra from the list. If all elements of the list will be removed, then the construction of noncomposite solids is finished. Otherwise, we pass to the next step: we construct the list of $(k+2)$-composite solids. 


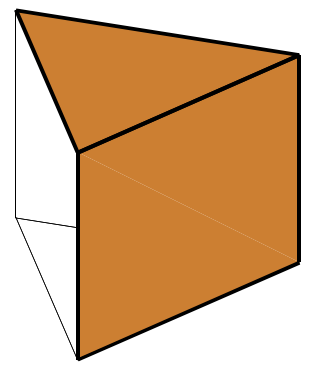

Figure $7 . \Pi_{3}$.

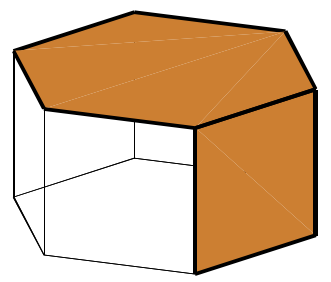

Figure $10 . \Pi_{6}$.

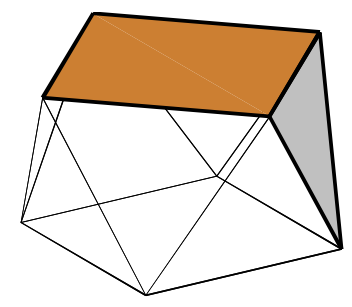

Figure 13. $A_{4}$.

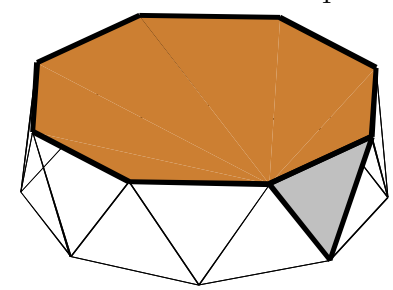

FiguRE 16. $A_{8}$.

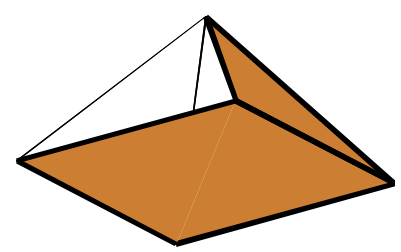

Figure 19. $M_{2}$.

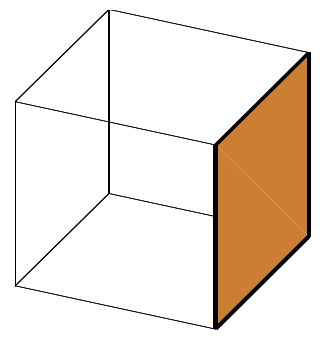

Figure 8. $[4,4,4]$.

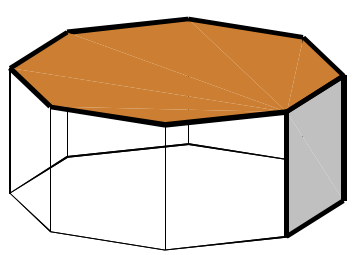

Figure 11. $\Pi_{8}$.

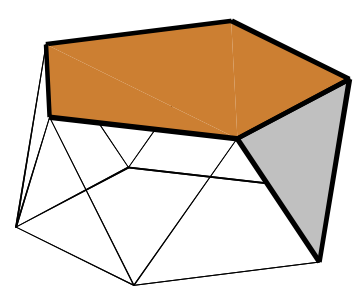

Figure 14. $A_{5}$.

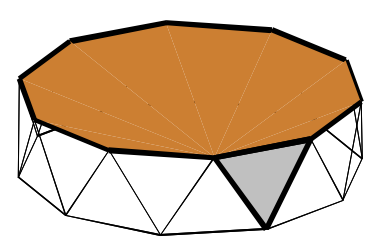

Figure $17 . A_{10}$.

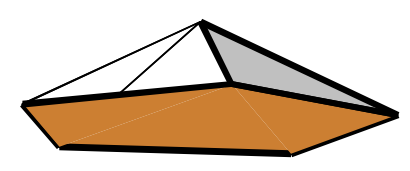

Figure 20. $M_{3}$.

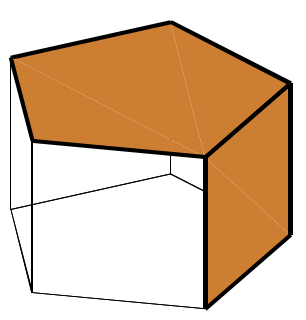

FiguRe $9 . \Pi_{5}$.

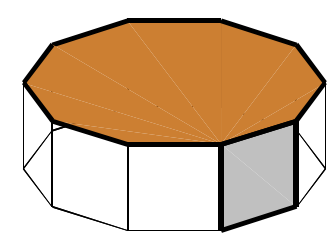

Figure $12 . \Pi_{10}$.

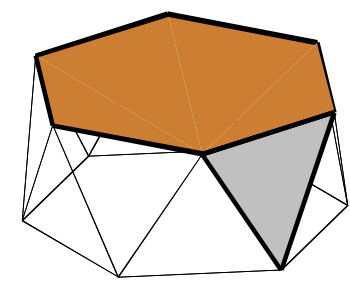

Figure 15. $A_{6}$.

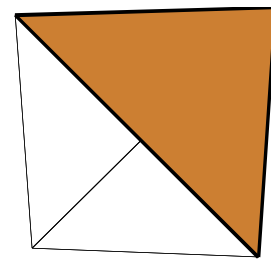

Figure 18. $M_{1}$.

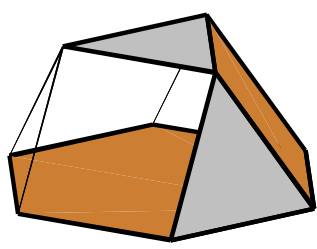

Figure 21. $M_{4}$. 


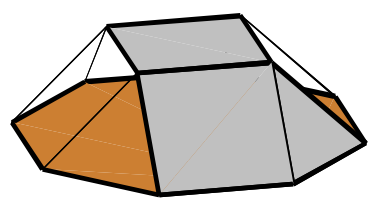

Figure $22 . M_{5}$.

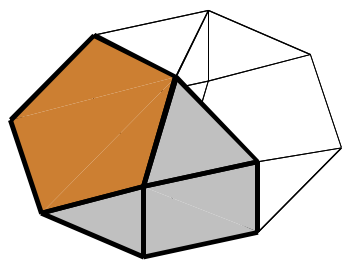

FiguRE 25. $M_{8}$.

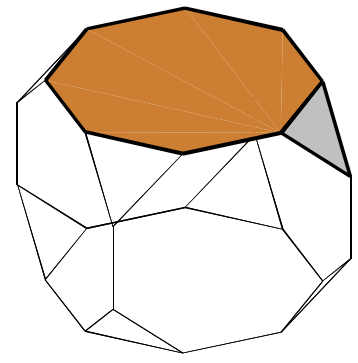

Figure 28. $M_{11}$.

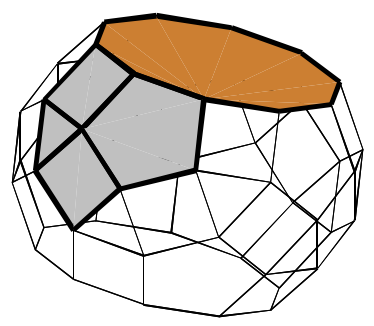

FIGURE $31 . M_{14}$.

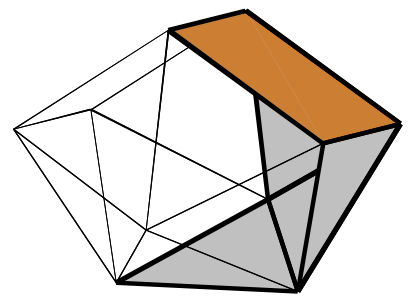

Figure $34 . M_{22}$.

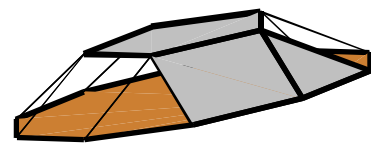

Figure $23 . M_{6}$.

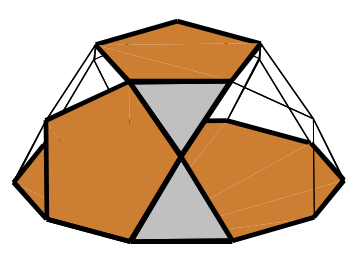

Figure 26. $M_{9}$.

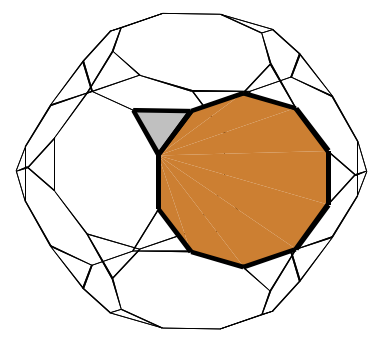

Figure 29. $M_{12}$.

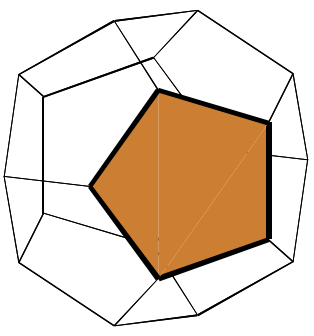

Figure $32 . M_{15}$.

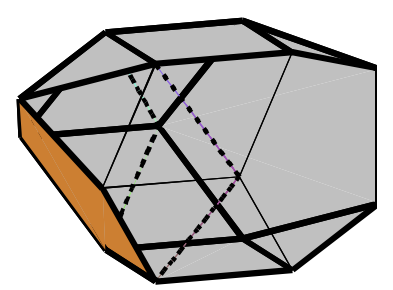

Figure $35 . Q_{4}$.

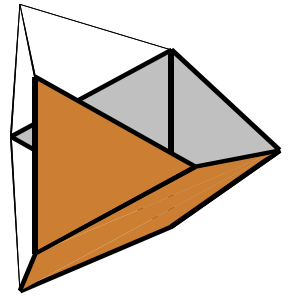

FiguRe $24 . M_{7}$.

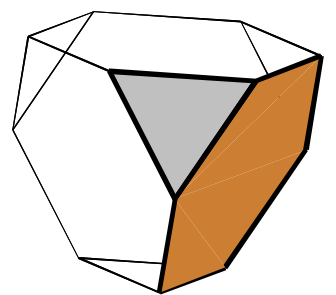

Figure 27. $M_{10}$.

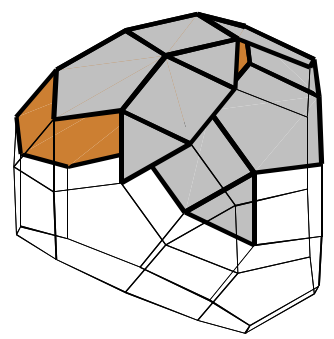

Figure $30 . M_{13}$.

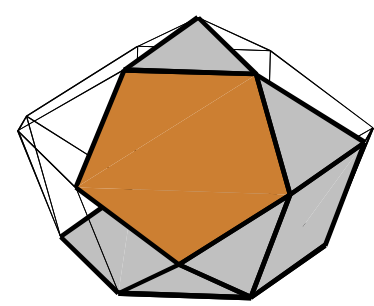

Figure 33. $M_{20}$.

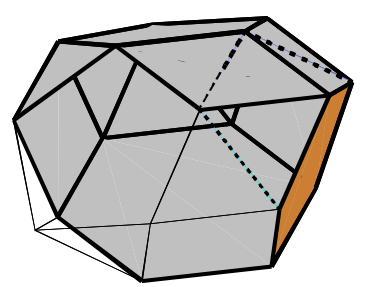

Figure $36 . Q_{6}$. 
Now we take a closer look at how to obtain all convex compositions of a convex regular-hedron and a noncomposite polyhedron.

Searching for all convex regular-hedra composed of a convex regular-hedron $P$ and a noncomposite polyhedron $Q$, we need to answer two questions: 1) will the polyhedron $P+Q$ be convex? and 2) are there other convex regular-hedra of the form $P+Q$ ?

Let $P$ be a convex regular-hedron, and $Q$ a noncomposite polyhedron. For obtaining all compositions of $P$ and $Q$ along their faces, it suffices to consider the pairs of equal superfundamental faces, one in $P$, and the other in $Q$. We denote one such pair by $\left(f_{P}, f_{Q}\right)$ and find all compositions of $P$ and $Q$ along $f_{P}$ and $f_{Q}$. Let $H_{P}$ be the maximal subgroup in $\operatorname{Aut}(P)$ such that the orbit $f_{P} H_{P}$ of the face $f_{P}$ consists of that face only. In accordance with the notation of $\$ 1$, the subgroup $H_{P}$ can be either the dihedral group $D_{2 \cdot k}$ of order $2 \cdot k$, where $k$ is a divisor of the number of sides of the face $f_{P}$, or the cyclic

TABle 2. Polyhedra $P_{1, k}$ with nonempty sets $S F$

\begin{tabular}{|r|l|c|l|}
\hline$k$ & Name & Notation & $S F$ \\
\hline 1 & Triangular prism & $\Pi_{3},[3,4,4]$ & 3,4 \\
\hline 2 & Cube & $\Pi_{4},[4,4,4]$ & 4 \\
\hline 3 & Pentagonal prism & $\Pi_{5},[4,4,5]$ & 4,5 \\
\hline 4 & Hexagonal prism & $\Pi_{6},[4,4,6]$ & 4,6 \\
\hline 5 & Octagonal prism & $\Pi_{8},[4,4,8]$ & 8 \\
\hline 6 & Decagonal prism & $\Pi_{10},[4,4,10]$ & 10 \\
\hline 7 & Square antiprism & $A_{4},[3,3,3,4]$ & 4 \\
\hline 8 & Pentagonal antiprism & $A_{5},[3,3,3,5]$ & 5 \\
\hline 9 & Hexagonal antiprism & $A_{6},[3,3,3,6]$ & 6 \\
\hline 10 & Octogonal antiprism & $A_{8},[3,3,3,8]$ & 8 \\
\hline 11 & Decagonal antiprism & $A_{10},[3,3,3,10]$ & 10 \\
\hline 12 & Tetrahedron & $M_{1},[3,3,3]$ & 3 \\
\hline 13 & Square pyramid & $M_{2}, J_{1}$ & 3,4 \\
\hline 14 & Pentagonal pyramid & $M_{3}, J_{2}$ & 5 \\
\hline 15 & Triangular cupola & $M_{4}, J_{3}$ & 4,6 \\
\hline 16 & Square cupola & $M_{5}, J_{4}$ & 8 \\
\hline 17 & Pentagonal cupola & $M_{6}, J_{5}$ & 10 \\
\hline 18 & Tridiminished icosahedron & $M_{7}, J_{63}$ & 3,5 \\
\hline 19 & Bilunabirotunda & $M_{8}, J_{91}$ & 5 \\
\hline 20 & Pentagonal rotunda & $M_{9}, J_{6}$ & $5,5,10$ \\
\hline 21 & Truncated tetrahedron & $M_{10},[3,6,6]$ & 6 \\
\hline 22 & Truncated cube & $M_{11},[3,8,8]$ & 8 \\
\hline 23 & Truncated dodecahedron & $M_{12},[3,10,10]$ & 10 \\
\hline 24 & $\begin{array}{l}\text { Tridiminished } \\
\text { rhombicosidodecahedron }\end{array}$ & $M_{13}, J_{83}$ & 10 \\
\hline 25 & $\begin{array}{l}\text { Parabidiminished } \\
\text { rhombicosidodecahedron }\end{array}$ & $M_{14}, J_{80}$ & 10 \\
\hline 26 & Dodecahedron & $M_{15},[5,5,5]$ & 5 \\
\hline 27 & $\begin{array}{l}\text { Triangular } \\
\text { hebesphenorotunda }\end{array}$ & $M_{20}, J_{92}$ & 5 \\
\hline 28 & Sphenocorona & $M_{22}, J_{86}$ & 4 \\
\hline 29 & Ivanov polyhedron & $Q_{4}$ & 5 \\
\hline 30 & Pryakhin polyhedron & 5 \\
\hline & & \\
\hline
\end{tabular}


group $C_{k}$ of rotations by multiples of $2 \pi / k$ about the axis orthogonal to the face $f_{P}$ and passing through the center of $f_{P}$. For example, $D_{2 \cdot 1}$ is the group of order 2 containing the reflection in the plane that orthogonally intersects the face $f_{P}$ by its symmetry axis.

If the face $f_{P}$ has a single fundamental edge under the action of the group $H_{P}$ on $P$, or the face $f_{Q}$ has a single fundamental edge under the action of the group $H_{Q}$, then the compositions of $P$ and $Q$ along the faces $f_{P}$ and $f_{Q}$ are all equal.

Otherwise, we consider one of the compositions $P+Q$ along the $m$-angled faces $f_{P}$, $f_{Q}$ and the group $D_{2 \cdot m}=\operatorname{Aut}\left(f_{P}\right)=\operatorname{Aut}\left(f_{Q}\right)$. Obviously, $H_{P}$ and $H_{Q}$ are subgroups of that group. If all edges of $f_{Q}$ are fundamental under the action of $H_{Q}$, or the same is true for $f_{P}$ and $H_{P}$, then every new composition of $P$ and $Q$ along $f_{P}, f_{Q}$ has the form $h P+Q$ with $h \in D_{2 \cdot m} \backslash H_{P}$, or the form $P+g Q$ with $g \in D_{2 \cdot m} \backslash H_{Q}$. Concerning the case where both subgroups $H_{P}, H_{Q}$ are nontrivial, now we only mention that the number of new compositions will be less than $2 \mathrm{~m}$.

We need to ensure definiteness for the actions prescribed by our algorithm of listing compositions of $P$ and $Q$ along entire faces. For this, it suffices, first, to order the set of superfundamental faces of each of the solids $P$ and $Q$; second, for every superfundamental face $f_{Q}$ of $Q$, to order the set of elements of the subgroup $H_{Q}$ of $\operatorname{Aut}(Q)$; and third, to order naturally the set of pairs of faces to be identified, and for a fixed pair $\left(f_{P}, f_{Q}\right)$ of $m$-angled faces, to define the order of listing of composition by the order of the elements of the set $D_{2 \cdot m} \backslash H_{Q}$ : we start with identifying the vertices with the smallest numbers, and the subsequent compositions are determined by the pairs $\left(f_{P}, h f_{Q}\right), h \in D_{2 \cdot m} \backslash H_{Q}$.

Sometimes, when searching compositions, we deviate from the above rule and change the order in the list of superfundamental faces, so as to make the notation shorter.

Clearly, our algorithm will output a list of all regular-hedra in the following order: all noncomposite polyhedra, all 2-composite polyhedra, all 3-composite polyhedra, ... .

We illustrate how the algorithm works by proving the following statement, in which the number of a list indicates the minimal number of noncomposite solids from which the polyhedrons on that list can be composed.

Proposition 5. Only the following composite regular-hedra can be obtained by composition of pentagonal pyramids $M_{3}$ and pentagonal rotundas $M_{9}$ :

2) $\mathrm{M}_{3}+\mathrm{M}_{3}, M_{3}+M_{9}, M_{3}+M_{9}^{\prime}, M_{9}+M_{9}, M_{9}+M_{9}^{\prime}$;

3) $M_{3}+M_{9}+M_{3}, M_{3}+M_{9}+M_{9}, M_{3}+M_{9}+M_{9}^{\prime}, M_{3}+M_{9}^{\prime}+M_{9}$;

4) $M_{3}+M_{9}+M_{3}+M_{9}, M_{3}+M_{9}+M_{3}+M_{9}^{\prime}, \mathrm{M}_{3}+\mathrm{M}_{9}+\mathrm{M}_{9}+\mathrm{M}_{3}, M_{3}+M_{9}+M_{9}^{\prime}+M_{3}$, $M_{3}+M_{9}+M_{9}^{\prime}+M_{3}^{\prime}, M_{3}+M_{9}+M_{9}^{\prime}+M_{3}^{\prime \prime}, \mathrm{M}_{3}+\mathrm{M}_{9}^{\prime}+\mathrm{M}_{9}+\mathrm{M}_{3}$

5) $\mathrm{M}_{3}+\mathrm{M}_{9}+\mathrm{M}_{3}+\mathrm{M}_{9}+\mathrm{M}_{3}, M_{3}+M_{9}+M_{3}+M_{9}^{\prime}+M_{3}, M_{3}+M_{9}+M_{3}+M_{9}^{\prime}+M_{3}^{\prime}$, $\mathrm{M}_{3}+\mathrm{M}_{9}+\mathrm{M}_{3}+\mathrm{M}_{9}^{\prime}+\mathrm{M}_{3}^{\prime \prime}$

6) $\mathrm{M}_{3}+\mathrm{M}_{9}+\mathrm{M}_{3}+\mathrm{M}_{9}^{\prime}+\mathrm{M}_{3}+\mathrm{M}_{3}$.

Proof. Observe that every triangular face of $M_{3}$ or $M_{9}$ has a vertex with curvature not exceeding $\pi / 3$. Since composition with a regular-hedron along triangular faces reduces the curvature of that vertex by at least $\pi / 3$, only pentagons and the decagon can be on the list of the superfundamental faces of the rotunda $M_{9}$ along which it can be enlarged up to a convex regular-hedron. For the pyramid $M_{3}$, such a list consists of the pentagon.

Obviously, $\operatorname{Aut}\left(M_{3}\right)=\operatorname{Aut}\left(M_{9}\right)=D_{2 \cdot 5}$. The fundamental faces of the pyramid $M_{3}$ are its pentagonal base and a triangle. The rotunda $M_{9}$ has five fundamental faces: the parallel 5-gon and 10-gon and two triangles. For each lateral face of the solids $M_{3}$ and $M_{9}$, the subgroup $H$ involved in our algorithm is the group $D_{2 \cdot 1}$, the only nontrivial symmetry in which is reflection in a plane. Therefore, the fundamental lateral triangular face has two fundamental edges under the action of $H$, and the fundamental lateral 5 -gon has 3 such edges. Each other fundamental face of the polyhedra $M_{3}$ and $M_{9}$ has a single fundamental edge, because the corresponding subgroup $H$ coincides with $D_{2.5}$. 
Lemma 3. All composite polyhedra that can be cut by a plane into noncomposite (possibly equal) solids of the form $M_{3}, M_{9}$ are on list 2) of Proposition 5 .

Proof. Composing pyramids $M_{3}$ results in a single regular-hedron, namely, the pentagonal bipyramid $M_{3}+M_{3}$; this is an $F$-polyhedron, and

$$
\mathrm{M}_{3}+\mathrm{M}_{3}=\mathrm{J}_{13} \text {. }
$$

The pyramid $M_{3}$ has one fundamental pentagonal face, and the rotunda $M_{9}$ has two such faces; each triangular face of any of these two solids makes at least two obtuse angles with faces adjacent to it. Therefore, one $M_{3}$ and one $M_{9}$ can give only two convex regular-hedra, which will be denoted by $M_{3}+M_{9}$ and $M_{3}+M_{9}^{\prime}$, and $\operatorname{Aut}\left(M_{3}+\right.$ $\left.M_{9}\right)=D_{2 \cdot 1}, \operatorname{Aut}\left(M_{3}+M_{9}^{\prime}\right)=D_{2 \cdot 5}$. We call the solid $\overline{M_{3}+M_{9}}$ the meta-augmented pentagonal rotunda (Figure 37) and the solid $\overline{M_{3}+M_{9}^{\prime}}$ the augmented pentagonal rotunda (Figure 38).

It remains to find the compositions of two rotundas. The angle between the decagonal face and a face adjacent to it is acute, and the angles between two adjacent faces that are not 10-gons are obtuse. Therefore, only compositions of two rotundas along decagonal faces can be convex. Such a face of $M_{9}$ has two fundamental edges that connect this face to a triangle and to a pentagon. Consequently, there exist at most two convex regularhedra obtained by composition of two rotundas $M_{9}$. Both of them are well known: the Archimedean solid $M_{9}+M_{9}$, i.e., the icosidodecahedron [3,5,3,5], and the pentagonal orthobirotunda $M_{9}+M_{9}^{\prime}=J_{34}$. Lemma 3 is proved.

Lemma 4. All 3-composite polyhedra obtained by composition of solids of the form $M_{3}$, $M_{9}$ are on list 3) of Proposition 5 .

Proof. On list 2) of Proposition 5, there are 4 regular-hedra denoted by non-sans-serif type letters:

$$
\overline{M_{3}+M_{9}}, \quad \overline{M_{3}+M_{9}^{\prime}}, \quad M_{9}+M_{9}, \quad M_{9}+M_{9}^{\prime} .
$$

We shall compose each of them with the pyramid $M_{3}$ and with the rotunda $M_{9}$.

The meta-augmented pentagonal rotunda $\overline{M_{3}+M_{9}}$ has three fundamental pentagonal faces, and compositions along two of them with the pyramid $M_{3}$ result in a trapezium-like face containing two conditional edges. Therefore, there is only one convex regular-hedron obtained by composition of the solids $\overline{M_{3}+M_{9}}$ and $M_{3}$, which will be denoted by

$$
\overline{M_{3}+M_{9}+M_{3}} \text {. }
$$

Figure 45 shows a composition of two such solids. The solids $\overline{M_{3}+M_{9}}$ and $M_{9}$ can only be composed along their decagonal faces to obtain a convex polyhedron. Since one of such faces has two fundamental edges, while the other has six, there are at most two convex compositions along these faces. Both of them do exist; we call the first the augmented icosidodecahedron (Figure 39) and the second the meta-augmented orthobirotunda (Figure 40), and denote them as follows:

$$
\overline{M_{3}+M_{9}+M_{9}} \text { and } \overline{M_{3}+M_{9}+M_{9}^{\prime}} \text {. }
$$

A convex composition of the solid $\overline{M_{3}+M_{9}^{\prime}}$ with the pyramid $M_{3}$ is not a regularhedron. Joining the polyhedron $\overline{M_{3}+M_{9}^{\prime}}$ and the rotunda $M_{9}$, we obtain either the augmented icosidodecahedron considered above, or a polyhedron to be called the elongated augmented orthobirotunda (Figure 41) and to be written as

$$
\overline{M_{3}+M_{9}^{\prime}+M_{9}} \text {. }
$$

If we compose any of the two polyhedra $M_{9}+M_{9}, M_{9}+M_{9}^{\prime}$ with the pyramid $M_{3}$ along pentagonal faces, then the result is one of the polyhedra considered above, and compositions with the rotunda $M_{9}$ lead to nonconvex polyhedra. 


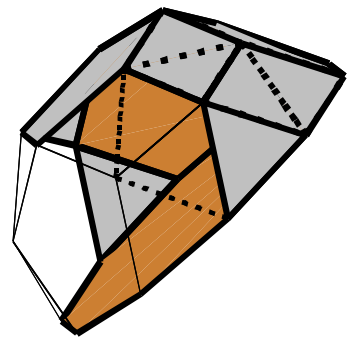

Figure $37 . P_{2,30}$.

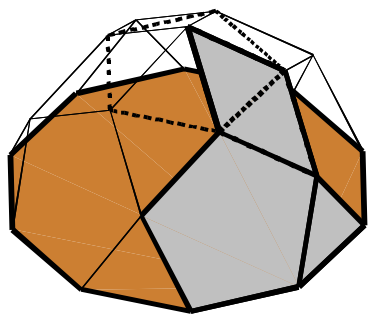

Figure $38 . P_{2,31}$.

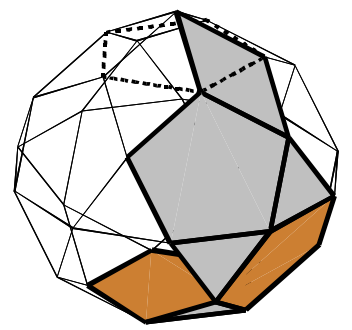

FiguRe $39 . P_{3,38}$.

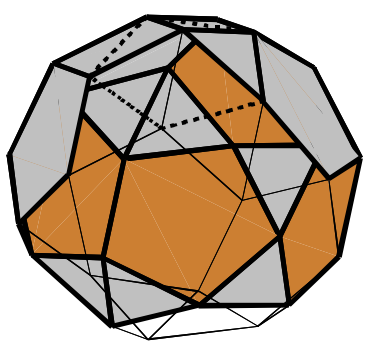

Figure $40 . P_{3,39}$.

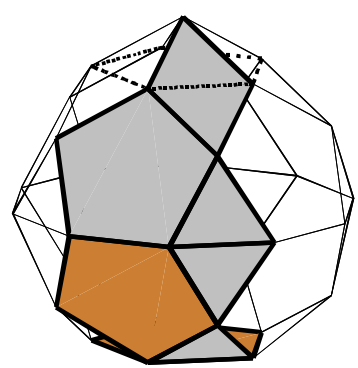

Figure 41. $P_{3,42}$.

Thus, composing three solids of the form $M_{3}, M_{9}$, we can obtain only four convex regular-hedra

$$
\overline{M_{3}+M_{9}+M_{3}}, \quad \overline{M_{3}+M_{9}+M_{9}}, \quad \overline{M_{3}+M_{9}+M_{9}^{\prime}}, \quad \overline{M_{3}+M_{9}^{\prime}+M_{9}} ;
$$

the groups of symmetries of the first and third of them are equal to $D_{2 \cdot 1}$, and for the other two solids this group is $D_{2 \cdot 5}$. Lemma 4 is proved.

Lemma 5. All 4-composite polyhedra composed of solids of the form $M_{3}, M_{9}$ are on list 4) of Proposition 5 .

Proof. We consider the compositions of each polyhedron in (3) with $M_{3}$ or $M_{9}$.

A convex composition of $\overline{M_{3}+M_{9}+M_{3}}$ and $M_{3}$ is not a regular-hedron. Composing the solids $\overline{M_{3}+M_{9}+M_{3}}$ and $M_{9}$, we can obtain exactly two convex regular-hedra, which we shall write as

$$
\overline{M_{3}+M_{9}+M_{3}+M_{9}}, \quad \overline{M_{3}+M_{9}+M_{3}+M_{9}^{\prime}},
$$

calling them the bimeta-augmented icosidodecahedron and the bi-augmented birotunda, respectively. Observe that

$$
\operatorname{Aut}\left(\overline{M_{3}+M_{9}+M_{3}+M_{9}}\right)=D_{2 \cdot 2}, \quad \operatorname{Aut}\left(\overline{M_{3}+M_{9}+M_{3}+M_{9}^{\prime}}\right)=D_{2 \cdot 1} .
$$

The compositions of the remaining three solids in (3) with the rotunda $M_{9}$ are nonconvex. Therefore, we compose each of them with the pyramid $M_{3}$. The compositions of $\overline{M_{3}+M_{9}+M_{9}}$ (Figure 39) with $M_{3}$ lead to a single new regular-hedron, which will be denoted by

$$
\overline{M_{3}+M_{9}+M_{9}+M_{3}}
$$

and called the parabi-augmented icosidodecahedron. This solid is an $F$-polyhedron.

If we compose the meta-augmented orthobirotunda $\overline{M_{3}+M_{9}+M_{9}^{\prime}}$ with the pyramid $M_{3}$ by joining its base with the pentagon of the second summand, then the result will be either the bimeta-augmented birotunda $\overline{M_{3}+M_{9}+M_{3}+M_{9}^{\prime}}$ treated before, or a solid that is not a regular-hedron. Therefore, we join the pyramid $M_{3}$ along its base 
with a pentagon of the 3 rd summand of the solid $\overline{M_{3}+M_{9}+M_{9}^{\prime}}$. Among four such compositions (we have four fundamental pentagons), three are regular-hedra. They are written as

$$
\overline{M_{3}+M_{9}+M_{9}^{\prime}+M_{3}}, \quad \overline{M_{3}+M_{9}+M_{9}^{\prime}+M_{3}^{\prime}}, \quad \overline{M_{3}+M_{9}+M_{9}^{\prime}+M_{3}^{\prime \prime}} .
$$

The first of them has adjacent rhombic faces, each of which is viewed as two triangular faces, and

$$
\begin{aligned}
& \operatorname{Aut}\left(\overline{M_{3}+M_{9}+M_{9}^{\prime}+M_{3}}\right)=\operatorname{Aut}\left(\overline{M_{3}+M_{9}+M_{9}^{\prime}+M_{3}^{\prime}}\right)=C_{2}, \\
& \operatorname{Aut}\left(\overline{M_{3}+M_{9}+M_{9}^{\prime}+M_{3}^{\prime \prime}}\right)=D_{2 \cdot 1} .
\end{aligned}
$$

In accordance with their order in (5), their projections are presented in Figures 42, 43, and 44. The summands occurring in the first two solids are depicted from right to left and from bottom to top, and for the third solid they are depicted from left to right and from bottom to top.

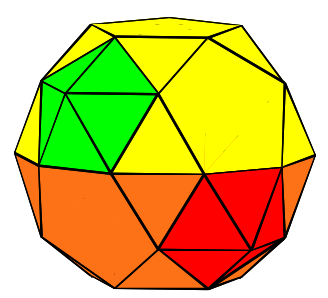

Figure 42. $P_{4,19}$.

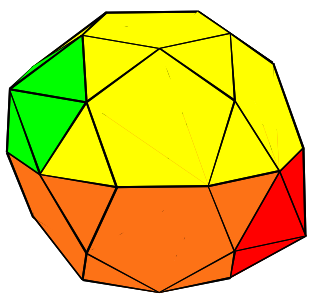

Figure $43 . P_{4,20}$.

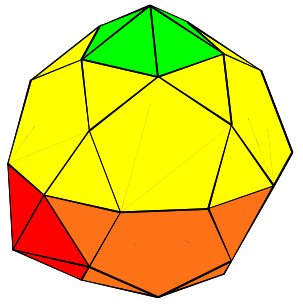

Figure 44. $P_{4,21}$.

The elongated augmented orthobirotunda $\overline{M_{3}+M_{9}^{\prime}+M_{9}}$ has exactly three fundamental pentagonal faces. However, composition along only one of them with the pyramid $M_{3}$ leads to a convex regular-hedron not treated before, which will be denoted by

$$
\overline{M_{3}+M_{9}^{\prime}+M_{9}+M_{3}}
$$

and will be called the bielongated orthobirotunda. It is an $F$-polyhedron. Lemma 5 is proved.

Lemma 6. All 5-composite polyhedra composed of solids of the form $M_{3}, M_{9}$ are on list 5) of Proposition 5 .

Proof. As was shown in the proof of Lemma 5 the composition of four solids of the form $M_{3}$ and $M_{9}$ results in exactly seven convex regular-hedra, and the five of them that are not $F$-polyhedra are listed in (4) and (5). We shall combine the pyramid $M_{3}$ with any polyhedron in (4) or (5), obtaining all convex regular-hedra composed of five solids of the form $M_{3}, M_{9}$.

The first polyhedron in (4) can be uniquely enlarged by the pyramid $M_{3}$ up to a convex regular-hedron. This results in a polyhedron to be called the tri-augmented icosidodecahedron and to be written as

$$
\overline{M_{3}+M_{9}+M_{3}+M_{9}+M_{3}} .
$$

Obviously, this is an $F$-polyhedron.

The second polyhedron in (4) has seven fundamental pentagonal faces, but compositions along only three of them with the pyramid $M_{3}$ are convex regular-hedra. We write them as

$$
\begin{array}{ll}
\overline{M_{3}+M_{9}+M_{3}+M_{9}^{\prime}+M_{3}}, & \overline{M_{3}+M_{9}+M_{3}+M_{9}^{\prime}+M_{3}^{\prime}}, \\
\overline{\mathrm{M}_{3}+\mathrm{M}_{9}+\mathrm{M}_{3}+\mathrm{M}_{9}^{\prime}+\mathrm{M}_{3}^{\prime \prime}} ; &
\end{array}
$$



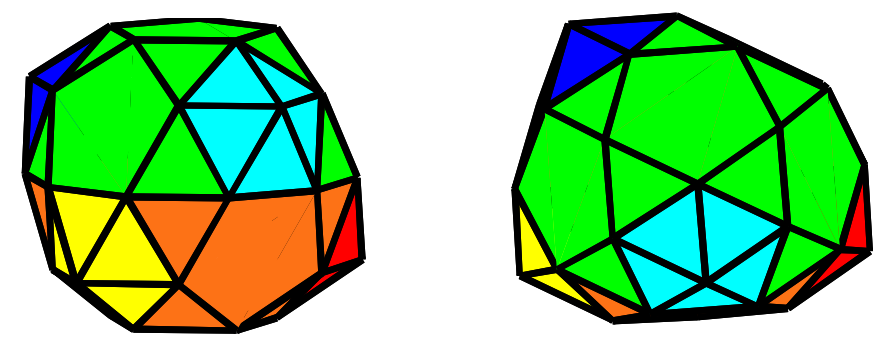

Figure 45. The 4-augmented pentagonal birotunda $\overline{M_{3}+M_{9}+M_{3}+M_{9}^{\prime}+M_{3}+M_{3}}$.

they can be distinguished by the number of pairs of rhombic faces: 2, 1, 0 , respectively. The third of these solids is an $F$-polyhedron.

Now we consider compositions of the pyramid $M_{3}$ with the polyhedra occurring on the list (5).

The first polyhedron on that list has five fundamental pentagonal faces, but compositions along only two of them are regular-hedra, and both occurred earlier in (6).

The polyhedron $\overline{M_{3}+M_{9}+M_{9}^{\prime}+M_{3}^{\prime}}$ also has five fundamental pentagonal faces, but only one of them can serve to composing with the pyramid $M_{3}$ to obtain a regular-

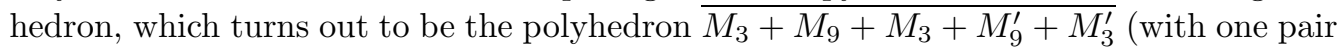
of rhombic faces).

Finally, the polyhedron $\overline{M_{3}+M_{9}+M_{9}^{\prime}+M_{3}^{\prime \prime}}$ has six fundamental pentagons, but the composition along only one of them with the pyramid $M_{3}$ is a regular-hedron. This latter is an $F$-polyhedron, to be denoted by $\overline{M_{3}+M_{9}+M_{3}+M_{9}^{\prime}+M_{3}^{\prime \prime}}$. Lemma 6 is proved.

Thus, we have proved that five solids of the form $M_{3}, M_{9}$ can be composed to give exactly four convex regular-hedra. Two of them, placed on the list (6), are not $F$-polyhedra. Compositions of each of them with $M_{3}$ result in only one regular-hedron, which will be written as

$$
\overline{M_{3}+M_{9}+M_{3}+M_{9}^{\prime}+M_{3}+M_{3}}
$$

and will be called the 4-augmented pentagonal birotunda. Since this is an $F$-polyhedron, we have constructed a list of all convex regular-hedra that are compositions of several solids $M_{3}$ and $M_{9}$.

Proposition 5 is proved.

Running ahead, we note that Proposition 5 will be needed in the proof of Theorem 1 . where the following statement will also be applied.

Proposition 6. Only the following composite regular-hedra can be obtained by composition of pentagonal cupolas $M_{6}$ and truncated dodecahedra $M_{12}$ :

2) $\mathrm{M}_{6}+\mathrm{M}_{6}, \mathrm{M}_{6}+\mathrm{M}_{6}^{\prime}, M_{6}+M_{12}, \overline{M_{6}+M_{12}^{\prime}}$;

3) $M_{6}+M_{12}+M_{6}, \overline{M_{6}+M_{12}+M_{6}^{\prime}}, \mathrm{M}_{6}+\mathrm{M}_{12}+\mathrm{M}_{6}^{\prime \prime}, \overline{\mathrm{M}_{6}+\mathrm{M}_{12}+\mathrm{M}_{6}^{\prime \prime \prime}}, \overline{M_{6}+M_{12}^{\prime}+M_{6}}$, and $\overline{\mathrm{M}_{6}+\mathrm{M}_{12}^{\prime}+\mathrm{M}_{6}^{\prime}}$;

4) $\quad M_{6}+M_{12}+M_{6}+M_{6}, \quad \overline{M_{6}+M_{12}+M_{6}^{\prime}+M_{6}}, \quad \overline{M_{6}+M_{12}+M_{6}^{\prime}+M_{6}^{\prime}}, \quad$ and $\overline{\mathrm{M}_{6}+\mathrm{M}_{12}^{\prime}+\mathrm{M}_{6}+\mathrm{M}_{6}}$,

where the F-polyhedra are written in sans-serif type letters.

Proof. Each vertex of the pentagonal face of the cupola $M_{6}$ (Figure 23) has curvature $12^{\circ}$. If a regular-hedron is adjoined along a face incident to such a vertex, then this curvature will reduce by at least $12^{\circ}$. Therefore, the cupola $M_{6}$ can be enlarged up 
to a regular-hedron only along its decagonal face. The truncated dodecahedron $M_{12}$ (Figure 29) possesses the same property, because the composition of it along a triangular face with the tetrahedron (and thereby, with any other noncomposite polyhedron) is a nonconvex polyhedron.

The enlarging of the cupola by itself yields two well-known polyhedra:

$$
M_{6}+M_{6}=J_{31}, \quad M_{6}+M_{6}^{\prime}=J_{30}
$$

If we compose the cupola $M_{6}$ with the solid $M_{12}$ so that two triangles become adjacent, then the angle between these triangular faces will be equal to $\pi$, i.e., the common edge of these faces will be conditional. Therefore, among the compositions of two different solids $M_{6}$ and $M_{12}$, one polyhedron is the Johnson solid $M_{6}+M_{12}=J_{68}$ (Figure 46), and the other has conditional edges. The latter solid will be denoted by $\overline{M_{6}+M_{12}^{\prime}}$ (Figure 47) and called the gyrate augmented truncated dodecahedron.

The first two of the four solids above are $F$-polyhedra. For each of the other two solids, the superfundamental faces are two decagonal faces.

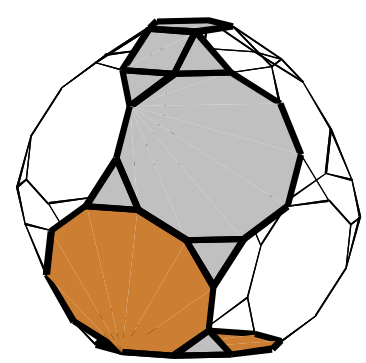

Figure $46 . P_{2,47}$.

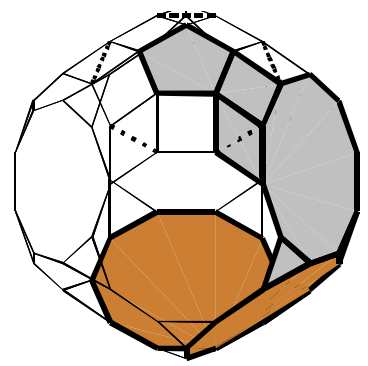

Figure $47 . P_{2,48}$.

The truncated dodecahedron $M_{12}$ is the equiangular-semiregular solid [3,10,10], and the pentagonal cupola $M_{6}$ is cut from the rhombicosidodecahedron $[3,4,5,4]$. These Archimedean solids are both obtained by a section of the dodecahedron; triangular faces arise in place of the vertices of the dodecahedron, and in the case of the polyhedron $[3,4,5,4]$ we have also square faces instead of the dodecahedron's edges. Naturally, these square faces make equal angles with the pentagonal faces of the solid $[3,4,5,4]$. Therefore, if two adjacent faces of $M_{12}$ are enlarged by two cupolas $M_{6}$ so that the two cupolas have a common edge of their square faces, then these squares will be in one plane, and the curvature at each of their common vertices will be zero.

The other compositions of $M_{12}$ with cupolas $M_{6}$ along adjacent faces are nonconvex. Consequently, we can obtain a convex regular-hedron only by adjoining cupolas $M_{6}$ along nonadjacent faces of $M_{12}$.

The augmented truncated dodecahedron $M_{6}+M_{12}$ can be enlarged by the rotunda $M_{6}$ up to only four convex regular-hedra, two of which are Johnson's well-known solids, and two need to be named:

$M_{6}+M_{12}+M_{6}=J_{70}$ is the metabi-augmented truncated dodecahedron,

$\overline{M_{6}+M_{12}+M_{6}^{\prime}}$ is the gyrate metabi-augmented truncated dodecahedron (Figure 48),

$\mathrm{M}_{6}+\mathrm{M}_{12}+\mathrm{M}_{6}^{\prime \prime}=\mathrm{J}_{69}$ is the parabi-augmented truncated dodecahedron, and

$\mathrm{M}_{6}+\mathrm{M}_{12}+\mathrm{M}_{6}^{\prime \prime \prime}$ is the gyrate parabi-augmented truncated dodecahedron.

The second and fourth of these polyhedra can also be obtained by enlarging the solid $\overline{M_{6}+M_{12}^{\prime}}$. There are only two enlargements of this solid by the cupola $M_{6}$ :

$$
\overline{M_{6}+M_{12}^{\prime}+M_{6}} \text { and } \overline{\mathrm{M}_{6}+\mathrm{M}_{12}^{\prime}+\mathrm{M}_{6}^{\prime}} \text {. }
$$




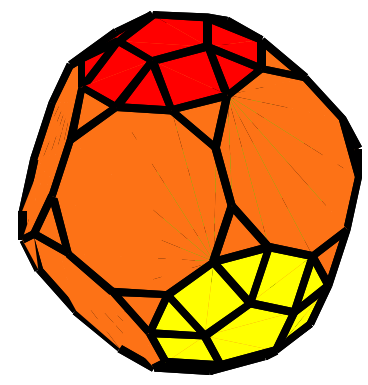

Figure 48. $\overline{M_{6}+M_{12}+M_{6}^{\prime}}$.

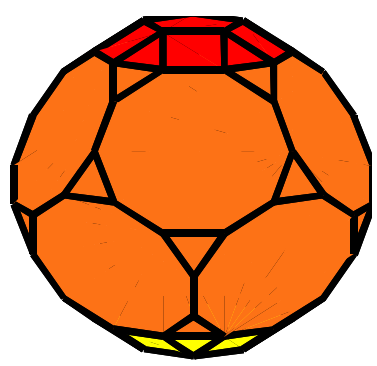

Figure $49 . \overline{\mathrm{M}_{6}+\mathrm{M}_{12}^{\prime}+\mathrm{M}_{6}^{\prime}}$.

We call them the bigyrate meta-augmented truncated dodecahedron and the bigyrate paraaugmented truncated dodecahedron, respectively.

Observe that truncated dodecahedra enlarged along opposite faces are $F$-polyhedra, and the number of superfundamental faces for each of the other three solids is equal to two. In the latter case, these two decagonal faces are symmetric to each other. The enlargements along any of these faces by the cupola $M_{6}$ lead to four $F$-polyhedra, one of which turns out to be the Johnson solid

$$
J_{71}=M_{6}+M_{12}+M_{6}+M_{6} .
$$

The other three polyhedra will be named and denoted as follows:

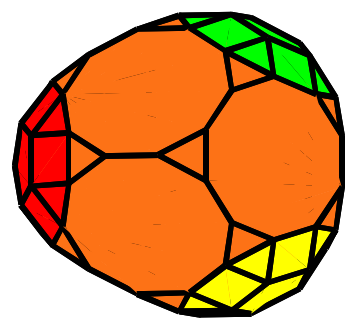

Figure 50. $\mathrm{P}_{4,26}$.

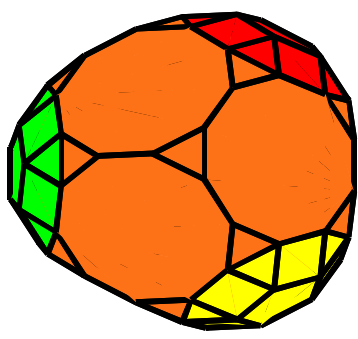

Figure 51. $P_{4,27}$.

\footnotetext{
$\overline{\mathrm{M}_{6}+\mathrm{M}_{12}+\mathrm{M}_{6}+\mathrm{M}_{6}^{\prime}}$ is the gyrate tri-augmented truncated dodecahedron,

$\mathrm{M}_{6}+\mathrm{M}_{12}+\mathrm{M}_{6}^{\prime}+\mathrm{M}_{6}$ is the bigyrate tri-augmented truncated dodecahedron (Figure 50 ),

$\overline{\mathrm{M}_{6}+\mathrm{M}_{12}^{\prime}+\mathrm{M}_{6}+\mathrm{M}_{6}}$ is the trigyrate augmented truncated dodecahedron.

Proposition 6 is proved.
} and

\section{§3. TheOrem on the Classification of NONCOMPosite Regular-HEDRA}

Theorem 1. A polyhedron is composite if and only if it is representable as one of the following compositions of noncomposite solids:

2) $\Pi_{3}+\Pi_{3}, \overline{\Pi_{3}+\Pi_{3}^{\prime}}, \overline{\Pi_{3}+\Pi_{4}}, \overline{\Pi_{3}+\Pi_{5}}, \Pi_{3}+M_{1}, \Pi_{3}+M_{2}, \Pi_{4}+M_{2}, \Pi_{5}+M_{2}$, $\Pi_{5}+M_{3}, \Pi_{6}+M_{2}, \Pi_{6}+M_{4}, \Pi_{8}+M_{5}, \Pi_{10}+M_{6}, \Pi_{10}+M_{9}, A_{4}+M_{2}, A_{5}+M_{3}$, $A_{6}+M_{4}, A_{8}+M_{5}, A_{10}+M_{6}, A_{10}+M_{9}, \mathrm{M}_{1}+\mathrm{M}_{1}, \overline{M_{1}+M_{2}}, \mathrm{M}_{1}+\mathrm{M}_{7}, M_{2}+M_{2}$, $\overline{M_{2}+M_{4}}, \mathrm{M}_{2}+\mathrm{M}_{22}, \mathrm{M}_{3}+\mathrm{M}_{3}, M_{3}+M_{7}, \overline{M_{3}+M_{8}}, \overline{M_{3}+M_{9}}, \overline{M_{3}+M_{9}^{\prime}}, M_{3}+M_{15}$, $\overline{\mathrm{M}_{3}+\mathrm{M}_{20}}, \overline{\mathrm{M}_{3}+\mathrm{Q}_{4}}, M_{4}+M_{4}, M_{4}+M_{4}^{\prime}, \mathrm{M}_{4}+\mathrm{M}_{10}, \overline{\mathrm{M}_{4}+\mathrm{M}_{10}^{\prime}}, \mathrm{M}_{5}+\mathrm{M}_{5}, \mathrm{M}_{5}+\mathrm{M}_{5}^{\prime}$, 
$M_{5}+M_{11}, \overline{M_{5}+M_{11}^{\prime}}, \mathrm{M}_{6}+\mathrm{M}_{6}, \mathrm{M}_{6}+\mathrm{M}_{6}^{\prime}, M_{6}+M_{9}, M_{6}+M_{9}^{\prime}, M_{6}+M_{12}, \overline{M_{6}+M_{12}^{\prime}}$, $M_{6}+M_{13}, M_{6}+M_{13}^{\prime}, M_{6}+M_{14}, M_{6}+M_{14}^{\prime}, M_{9}+M_{9}, M_{9}+M_{9}^{\prime} ;$

3) $\overline{P_{2,2}+M_{2}}, \overline{\mathrm{P}_{2,3}+\Pi_{3}}, \overline{\mathrm{P}_{2,3}+\mathrm{M}_{2}}, \overline{\mathrm{P}_{2,4}+\mathrm{M}_{2}}, \mathrm{P}_{2,5}+\mathrm{M}_{1}, P_{2,6}+M_{2}, \mathrm{P}_{2,7}+\mathrm{M}_{2}$, $\mathrm{P}_{2,8}+\mathrm{M}_{2}, \mathrm{P}_{2,9}+\mathrm{M}_{3}, P_{2,10}+M_{2}, \mathrm{P}_{2,10}+\mathrm{M}_{2}^{\prime}, \mathrm{P}_{2,11}+\mathrm{M}_{4}, \mathrm{P}_{2,11}+\mathrm{M}_{4}^{\prime}, \mathrm{P}_{2,12}+\mathrm{M}_{5}, \mathrm{P}_{2,12}+$ $\mathrm{M}_{5}^{\prime}, \mathrm{P}_{2,13}+\mathrm{M}_{6}, \mathrm{P}_{2,13}+\mathrm{M}_{6}^{\prime}, P_{2,13}+M_{9}, P_{2,13}+M_{9}^{\prime}, \overline{P_{2,14}+M_{3}}, P_{2,14}+M_{9}, P_{2,14}+M_{9}^{\prime}$, $\mathrm{P}_{2,15}+\mathrm{M}_{2}, \mathrm{P}_{2,16}+\mathrm{M}_{3}, \mathrm{P}_{2,17}+\mathrm{M}_{4}, \mathrm{P}_{2,18}+\mathrm{M}_{5}, \mathrm{P}_{2,19}+\mathrm{M}_{6}, P_{2,19}+M_{9}, \overline{P_{2,20}+M_{3}}$, $P_{2,20}+M_{9}, \overline{P_{2,22}+M_{2}}, \overline{P_{2,25}+M_{4}}, \overline{P_{2,25}+M_{4}^{\prime}}, \overline{\mathrm{P}_{2,29}+\mathrm{M}_{3}}, \overline{P_{2,30}+M_{3}}, \overline{P_{2,30}+M_{6}}$, $\overline{P_{2,30}+M_{6}^{\prime}}, \overline{P_{2,30}+M_{9}}, \overline{P_{2,30}+M_{9}^{\prime}}, \overline{\mathrm{P}_{2,31}+\mathrm{M}_{6}}, \overline{\mathrm{P}_{2,31}+\mathrm{M}_{6}^{\prime}}, \overline{P_{2,31}+M_{9}}, P_{2,32}+M_{3}$, $\mathrm{P}_{2,32}+\mathrm{M}_{3}^{\prime}, \mathrm{P}_{2,41}+\mathrm{M}_{5}, \overline{\mathrm{P}_{2,41}+\mathrm{M}_{5}^{\prime}}, \overline{\mathrm{P}_{2,42}+\mathrm{M}_{5}}, P_{2,47}+M_{6}, \overline{P_{2,47}+M_{6}^{\prime}}, \mathrm{P}_{2,47}+\mathrm{M}_{6}^{\prime \prime}$, $\overline{\mathrm{P}_{2,47}+\mathrm{M}_{6}^{\prime \prime \prime}}, \overline{P_{2,48}+M_{6}}, \overline{\mathrm{P}_{2,48}+\mathrm{M}_{6}^{\prime}}, P_{2,49}+M_{6}, P_{2,50}+M_{6}, \mathrm{P}_{2,51}+\mathrm{M}_{6}, \mathrm{P}_{2,51}+\mathrm{M}_{6}^{\prime}$, $\mathrm{P}_{2,52}+\mathrm{M}_{6}$

4) $\overline{\mathrm{P}_{3,1}+\mathrm{M}_{2}}, \overline{\mathrm{P}_{3,1}+\mathrm{M}_{2}^{\prime}}, \mathrm{P}_{3,6}+\mathrm{M}_{2}, \mathrm{P}_{3,10}+\mathrm{M}_{2}, \overline{\mathrm{P}_{3,18}+\mathrm{M}_{3}}, \overline{\mathrm{P}_{3,19}+\mathrm{M}_{3}}, \overline{P_{3,20}+M_{9}}$, $\overline{P_{3,20}+M_{9}^{\prime}}, \overline{\mathrm{P}_{3,28}+\mathrm{M}_{3}}, \overline{P_{3,29}+M_{9}}, \overline{\mathrm{P}_{3,31}+\mathrm{M}_{1}}, \overline{\mathrm{P}_{3,32}+\mathrm{M}_{2}}, \overline{\mathrm{P}_{3,33}+\mathrm{M}_{2}}, \overline{\mathrm{P}_{3,35}+\mathrm{M}_{6}}$, $\overline{\mathrm{P}_{3,35}+\mathrm{M}_{6}^{\prime}}, \overline{P_{3,35}+M_{9}}, \overline{P_{3,35}+M_{9}^{\prime}}, \overline{\mathrm{P}_{3,38}+\mathrm{M}_{3}}, \overline{P_{3,39}+M_{3}}, \overline{P_{3,39}+M_{3}^{\prime}}, \overline{P_{3,39}+M_{3}^{\prime \prime}}$, $\overline{\mathrm{P}_{3,42}+\mathrm{M}_{3}}, \quad \mathrm{P}_{3,43}+\mathrm{M}_{3}, \mathrm{P}_{3,48}+\mathrm{M}_{6}, \overline{\mathrm{P}_{3,49}+\mathrm{M}_{6}}, \overline{\mathrm{P}_{3,49}+\mathrm{M}_{6}^{\prime}}, \overline{\mathrm{P}_{3,52}+\mathrm{M}_{6}}, \quad \mathrm{P}_{3,54}+\mathrm{M}_{6}$, $\mathrm{P}_{3,55}+\mathrm{M}_{6}$

5) $\overline{\mathrm{P}_{4,7}+\mathrm{M}_{3}}, \overline{\mathrm{P}_{4,8}+\mathrm{M}_{3}}, \overline{\mathrm{P}_{4,10}+\mathrm{M}_{3}}, \overline{\mathrm{P}_{4,16}+\mathrm{M}_{3}}, \overline{P_{4,17}+M_{3}}, \overline{P_{4,17}+M_{3}^{\prime}}, \overline{\mathrm{P}_{4,17}+\mathrm{M}_{3}^{\prime \prime}}$;

6) $\overline{P_{5,5}+M_{3}}$.

The number of noncomposite summands in each composition above is minimal, and the index $i$ of the polyhedron $P_{k, i}$ indicates its number on the $k$ th list, $k=2,3,4,5,6$.

Proof. We shall use the algorithm for the synthesis of convex regular-hedra, as described above, to construct all $k$-composite polyhedra, $k=2,3, \ldots$ We include in tables those of them that possess at least one superfundamental face. The names of these polyhedra, the Plato, Archimedes, and Johnson solids that are absent in our tables, the $F$-polyhedra with conditional edges, and also drawings will allow the reader to establish a one-to-one correspondence between the polyhedra and the formulas describing them. Then, the proof of the theorem reduces to checking the outputs of the algorithm, as this was done in the proof of Propositions 5 and 6 .

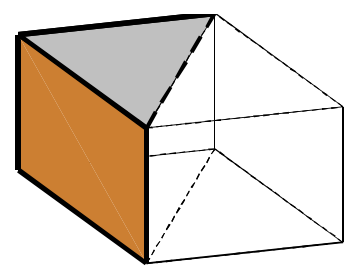

Figure $52 . \mathrm{P}_{2,2}$.

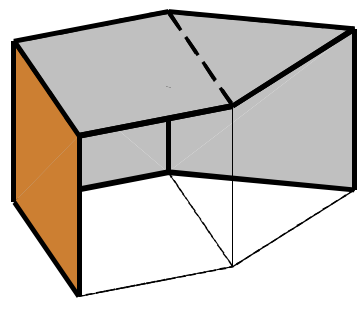

FIGURE 53. $\mathrm{P}_{2,3}$.

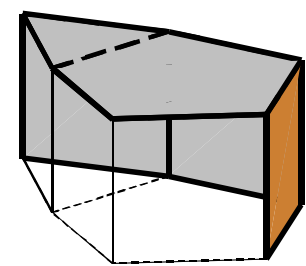

FiguRE 54. $\mathrm{P}_{2,4}$.

3.1. 2-composite polyhedra. Consider all convex regular-hedra obtained by composition of each noncomposite polyhedron of Table 2 with itself and with a polyhedron that stands below it in Table2. The resulting 2-composite solids will be denoted by $P_{2, i}, i=1$, $2, \ldots$, in the order of their arising. If a 2-composite polyhedron has a superfundamental face, we place it in Table 3 . Off the table are the $F$-polyhedra, the names of some of them, and the drawings.

The triangular prism $P_{1,1}=\Pi_{3}$ (Figure 7) has two superfundamental faces. Joining such prisms along square faces, we obtain two composite solids:

$$
\mathrm{P}_{2,1}=\Pi_{3}+\Pi_{3}=\mathrm{J}_{26} \text { and } P_{2,2} \text { (Figure 52). }
$$


The set of fundamental faces of each of them consists of a triangle and a square. The gyrobifastigium $\mathrm{P}_{2,1}$ is an $F$-polyhedron, because every triangular face of it makes an angle of $150^{\circ}$ with one of the adjacent squares, and it is orthogonal to the other two squares.

TABLE 3 . Polyhedra $P_{2, k}$ with nonempty sets $S F$

\begin{tabular}{|c|c|c|c|}
\hline$k$ & Name & Notation & $S F$ \\
\hline 2 & Rhombic prism & $\Pi_{3}+\Pi_{3}^{\prime}$ & 4 \\
\hline 3 & Augmented cube & $\Pi_{3}+\Pi_{4}$ & 4 \\
\hline 4 & Nonregular hexagonal prism & $\Pi_{3}+\Pi_{5}$ & 4 \\
\hline 5 & Elongated triangular pyramid & $\Pi_{3}+M_{1}, J_{7}$ & 3 \\
\hline 6 & Augmented triangular prism & $\Pi_{3}+M_{2}, J_{49}$ & 4 \\
\hline 7 & Elongated square pyramid & $\Pi_{4}+M_{2}, J_{8}$ & 4 \\
\hline 8 & Augmented pentagonal prism & $\Pi_{5}+M_{2}, J_{52}$ & 4 \\
\hline 9 & Elongated pentagonal pyramid & $\Pi_{5}+M_{3}, J_{9}$ & 5 \\
\hline 10 & Augmented hexagonal prism & $\Pi_{6}+M_{2}, J_{54}$ & 4,4 \\
\hline 11 & Elongated triangular cupola & $\Pi_{6}+M_{4}, J_{18}$ & 6 \\
\hline 12 & Elongated square cupola & $\Pi_{8}+M_{5}, J_{19}$ & 8 \\
\hline 13 & Elongated pentagonal cupola & $\Pi_{10}+M_{6}, J_{20}$ & 10 \\
\hline 14 & Elongated pentagonal rotunda & $\Pi_{10}+M_{9}, J_{21}$ & 5,10 \\
\hline 15 & $\begin{array}{l}\text { Gyroelongated square } \\
\text { pyramid }\end{array}$ & $A_{4}+M_{2}, J_{10}$ & 5 \\
\hline 16 & $\begin{array}{l}\text { Cyroelongated pentagonal } \\
\text { pyramid }\end{array}$ & $A_{5}+M_{3}, J_{11}$ & 4,6 \\
\hline 17 & $\begin{array}{l}\text { Gyroelongated triangular } \\
\text { cupola }\end{array}$ & $A_{6}+M_{4}, J_{22}$ & 8 \\
\hline 18 & $\begin{array}{l}\begin{array}{l}\text { Gyroelongated square } \\
\text { cupola }\end{array} \\
\end{array}$ & $A_{8}+M_{5}, J_{23}$ & 10 \\
\hline 19 & $\begin{array}{l}\text { Gyroelongated pentagonal } \\
\text { cupola }\end{array}$ & $A_{10}+M_{6}, J_{24}$ & 3,5 \\
\hline 20 & $\begin{array}{l}\text { Gyroelongated pentagonal } \\
\text { rotunda }\end{array}$ & $A_{10}+M_{9}, J_{25}$ & 5 \\
\hline 22 & Augmented square pyramid & $M_{1}+M_{2}$ & 4 \\
\hline 24 & Octahedron & $M_{2}+M_{2},[3,3,3,3]$ & 3 \\
\hline 25 & Augmented triangular cupola & $M_{2}+M_{4}$ & 6 \\
\hline 28 & Metabidiminished icosahedron & $M_{3}+M_{7}, J_{62}$ & 5 \\
\hline 29 & Augmented bilunabirotunda & $M_{3}+M_{8}$ & 5,5 \\
\hline 30 & $\begin{array}{l}\text { Meta-augmented } \\
\text { pentagonal rotunda }\end{array}$ & $M_{3}+M_{9}$ & 5,10 \\
\hline 31 & Augmented pentagonal rotunda & $M_{3}+M_{9}^{\prime}$ & 10 \\
\hline 32 & Augmented dodecahedron & $M_{3}+M_{15}, J_{58}$ & 5,5 \\
\hline 35 & Triangular orthobicupola & $M_{4}+M_{4}, J_{27}$ & 4 \\
\hline 36 & Cuboctahedron & $M_{4}+M_{4}^{\prime},[3,4,3,4]$ & 4 \\
\hline 41 & Augmented truncated cube & $M_{5}+M_{11}, J_{66}$ & 8 \\
\hline 42 & Elongated truncated cube & $M_{5}+M_{11}^{\prime}$ & 8 \\
\hline 45 & Pentagonal gyrocupolarotunda & $M_{6}+M_{9}, J_{33}$ & 5 \\
\hline 46 & \begin{tabular}{|l} 
Pentagonal orthocupolarotunda \\
\end{tabular} & $M_{6}+M_{9}^{\prime}, J_{32}$ & 5 \\
\hline
\end{tabular}




\begin{tabular}{|r|l|c|l|}
\hline$k$ & Name & Notation & $S F$ \\
\hline 47 & $\begin{array}{l}\text { Augmented truncated } \\
\text { dodecahedron }\end{array}$ & $M_{6}+M_{12}, J_{68}$ & 10,10 \\
\hline 48 & $\begin{array}{l}\text { Gyrate augmented } \\
\text { truncated dodecahedron }\end{array}$ & $M_{6}+M_{12}^{\prime}$ & 10,10 \\
\hline 49 & $\begin{array}{l}\text { Metabidiminished } \\
\text { rhombicosidodecahedron }\end{array}$ & $M_{6}+M_{13}, J_{81}$ & 10 \\
\hline 50 & $\begin{array}{l}\text { Gyrate bidiminished } \\
\text { rhombicosidodecahedron }\end{array}$ & $M_{6}+M_{13}^{\prime}, J_{82}$ & 10 \\
\hline 51 & Diminished rhombicosidodecahedron & $M_{6}+M_{14}, J_{76}$ & 10 \\
\hline 52 & $\begin{array}{l}\text { Paragyrate diminished } \\
\text { rhombicosidodecahedron }\end{array}$ & $M_{6}+M_{14}^{\prime}, J_{77}$ & 10 \\
\hline 53 & Icosidodecahedron & $M_{9}+M_{9},[3,5,3,5]$ & 5 \\
\hline 54 & Pentagonal orthobirotunda & $M_{9}+M_{9}^{\prime}, J_{34}$ & 5,5 \\
\hline
\end{tabular}

One prism $\Pi_{3}$ and one cube (Figure 8) yield only one composite polyhedron $P_{2,3}$ (Figure 53). The prism $\Pi_{5}$ (Figure 91) has two fundamental faces: a square and a pentagon. Only one composition of $\Pi_{3}$ and $\Pi_{5}$ is a convex regular-hedron; this is $P_{2,4}$ (Figure 54).

Composing the prism $\Pi_{3}$ with every prism (Figures 10-12) or antiprism (Figures 1317) standing below $\Pi_{5}$ in Table 2 , we do not get any regular-hedra.

The tetrahedron $M_{1}$ (Figure 18) has one fundamental face. Composition along it with the prism $\Pi_{3}$ results in the elongated triangular pyramid $P_{2,5}$. The square pyramid $M_{2}$ has two fundamental faces: a triangle and a square. Since the lateral faces adjacent to the triangle make obtuse angles with it, a convex regular-hedron can be obtained only by joining $\Pi_{3}$ and $M_{2}$ along the square faces. There is a unique such polyhedron $P_{2,6}$.

Every square or triangular face of any polyhedron placed after $M_{2}$ in Proposition 4 makes an obtuse angle with any adjacent face except, possibly, one of them. Consequently, we have considered all convex regular-hedra composed of the triangular prism and a noncomposite solid.

The composition of the cube $\Pi_{4}$ with itself, as well as that of any two prisms along their bases, is not a regular-hedron. Any polyhedron listed after $\Pi_{4}$ in Proposition 4 , except for the square pyramid $M_{2}$, has a square face that makes an obtuse angle with at least one face adjacent to it. Composing one cube and one pyramid, we get a single convex regular-hedron $P_{2,7}$.

With the exception of the pyramids $M_{2}$ and $M_{3}$, each square or pentagonal face of a polyhedron that stands below the pentagonal prism $\Pi_{5}$ in Table 2 makes an obtuse angle with some face adjacent of it. The exceptions mentioned above lead to the Johnson solids $P_{2,8}$ and $P_{2,9}$.

The angle between two adjacent lateral faces of the prism $\Pi_{8}$ (Figure 11), and also of any prism with a greater number of base edges, is so large that, even if we compose $\Pi_{8}$ along a lateral face with the pyramid $M_{2}$, we get a nonconvex polyhedron. Among the polyhedra of Proposition 4, only the square cupola $M_{5}$ has an octagonal face that makes acute angles with any face adjacent to it. Therefore, composing one prism $\Pi_{8}$ and one cupola $M_{5}$, we can obtain a single convex regular-hedron $P_{2,12}$.

Enlargements of the decagonal prism $\Pi_{10}$ (Figure 12) lead to only two regular-hedra $P_{2,13}$ and $P_{2,14}$.

Each antiprism $A_{m}, m=4,5,6,8,10$ (Figures 13-17), has two fundamental faces: an $m$-gon and a triangle. The angle between adjacent triangles grows with the number of sides of the base of the antiprism; already for the square antiprism this angle becomes so large that the adjoining to the antiprism's lateral face of any noncomposite solid 
yields a nonconvex polyhedron. Therefore, we are left with searching, in Proposition 4, the polyhedra that have an $m$-gonal face making an acute angle with any face adjacent to it. Only the solids $M_{2}, M_{3}, M_{4}, M_{5}, M_{6}, M_{9}$ have this property. By composing one of these solids with one prism $A_{n}$, we can only get the convex regular-hedra $P_{2, k}$, $k=15,16, \ldots, 20$.

The composition of the tetrahedron $M_{1}$ with itself is the triangular dipyramid

$$
\mathrm{P}_{2,21}=\mathrm{M}_{1}+\mathrm{M}_{1}=\mathrm{J}_{12} \text {, }
$$

and we cannot enlarge it up to a convex regular-hedron along its single fundamental face. Indeed, the angle between two adjacent faces of the tetrahedron exceeds $60^{\circ}$, and in the composition of three tetrahedra this angle will be tripled. So, the solid $\mathrm{P}_{2,21}$ is an $F$-polyhedron. Obviously, this enlargement of the pentagonal pyramid $M_{3}$ does not lead to a convex polyhedron. The polyhedra $M_{4}, M_{5}, M_{6}$, which follow $M_{3}$ in Proposition 4 (or Table 2), are obtained by cuts of the cube and the dodecahedron. Their triangular faces arose as a result of cutting off pyramids with the vertex plane angles equal to $90^{\circ}$ and $108^{\circ}$, respectively. Therefore, adjoining the pyramid $M_{3}$ (with the vertex plane angle equal to $60^{\circ}$ ) along such faces yields a nonconvex polyhedron. As a result of cutting off pyramids, we get the triangular faces of the solids $M_{9}, M_{11}$, $M_{12}, M_{13}, M_{14}$, and also (see [6]) the triangular faces of $M_{8}$ and $M_{20}$ that contain the vertex of type $[3,4,5,4]$. Therefore, no composition of these solids with the tetrahedron $M_{1}$ along the faces indicated, and no composition of $M_{1}$ with any polyhedron $M_{k}, k=$ $3,4,5,6,9,11,12,13,14$, is a convex regular-hedron. The enlargement of a tetrahedron by the square pyramid $M_{2}$ is possible only along a lateral face of $M_{2}$; this leads to $P_{2,22}$.

The composition of the tetrahedron $M_{1}$ and the tridiminished icosahedron along the face adjacent to three pentagons is the augmented tridiminished icosahedron

$$
P_{2,23}=M_{1}+M_{7}=J_{64}
$$

The adjoining of the tetrahedron to other faces of $M_{7}$ gives us a nonconvex polyhedron, because the other triangular faces of $M_{7}$ have adjacent triangular faces, the angle between which is equal to that between adjacent lateral faces of the pyramid $M_{3}$; see above. We prove that $P_{2,23}$ is an $F$-polyhedron. Indeed, we cannot adjoin a noncomposite polyhedron to a triangular face of $P_{2,23}$ without violating convexity. Enlargement along a single fundamental pentagon of the solid $P_{2,23}$ by a noncomposite polyhedron leads to a nonconvex polyhedron, because the angle between that pentagon and the adjacent triangle of the joined tetrahedron differs from $180^{\circ}$ by less than $10^{\circ}$, while for any noncomposite polyhedron the minimal angle between adjacent pentagonal and triangular faces exceeds $10^{\circ}$.

In Figure 25, the fundamental faces of the polyhedron $M_{8}$ are shadowed. Each of the two fundamental triangles has a vertex incident to two triangles, a square, and a pentagon. If we enlarge the solid $M_{8}$ by the tetrahedron $M_{1}$, then the curvature of that vertex becomes negative. Hence, we cannot obtain a regular-hedron by composing $M_{1}$ and $M_{8}$.

The dodecahedron $M_{15}$ has no triangular faces. Therefore, it is impossible to compose $M_{15}$ and $M_{1}$ to get a regular-hedron.

Above it was shown that no regular-hedron can be obtained by composing the tetrahedron and the solid $M_{20}$ along any of its triangular faces incident to a vertex of type $[3,5,3,5]$. As was proved in [4] (see also [7]), two fundamental triangles of $M_{20}$, i.e., the adjacent triangles shadowed in Figure 33, are arranged precisely as two adjacent triangles of the pentagonal pyramid $M_{3}$; consequently, adjoining the tetrahedron along such faces does not give a convex regular-hedron. 
Each triangular face of the sphenocorona $M_{22}$ (Figure 34) has a vertex incident to five edges. Therefore, we cannot get a regular-hedron by composing $M_{1}$ and $M_{22}$.

The construction of the Ivanov solid $Q_{4}$ (see Figure 35] and [3, 7]) shows that the triangular faces of $Q_{4}$ and the faces adjacent to them are arranged precisely as is the bilunabirotunda $M_{8}$. Hence, there are no convex compositions of $M_{1}$ and $Q_{4}$.

It remains to consider the compositions of $M_{1}$ and $Q_{6}$. The Pryakhin solid $Q_{6}$ (Figure 36) has four fundamental triangles. Each of them is located precisely as such triangles are located in the polyhedron $M_{20}$; see [7. Consequently, we cannot enlarge $Q_{6}$ by a tetrahedron to obtain a convex regular-hedron.

The composition of two pyramids $M_{2}$ along lateral faces will not be convex, because we saw that already the composition of the pyramids $M_{1}$ and $M_{2}$ is the regular-hedron $P_{2,22}$ with a conditional edge. The only convex regular-hedron obtained by composing two square pyramids is the octahedron $P_{2,24}$. Above it was proved that, except for the solid $M_{7}$, no polyhedron that stands in Proposition 4 to the right of $M_{2}$ can be enlarged up to a convex regular-hedron by a tetrahedron. Consequently, along a triangular face, such a polyhedron cannot be enlarged also by the pyramid $M_{2}$, in which the angle between two adjacent lateral faces is greater than a similar angle in the tetrahedron. If we join a lateral side of $M_{3}$ with the triangular face of the solid $M_{7}$ adjacent to three pentagons, then we get a vertex with negative curvature. Thus, it only remains to analyze the compositions along square faces of the pyramid $M_{2}$ with all polyhedra that stand in Proposition 4 to the right of $M_{2}$.

The square cupola $M_{4}$ has one fundamental square face. Composing $M_{4}$ along this face with the pyramid $M_{2}$, we get a convex regular-hedron $P_{2,25}$. The adjoining of the pyramid $M_{2}$ to each of the solids $M_{5}, M_{6}, M_{13}, M_{14}, M_{20}, Q_{6}$ along square faces results in the arising of a vertex with nonpositive curvature. The polyhedra $M_{7}, M_{9}, M_{10}, M_{11}$, $M_{12}, M_{15}$ have no square faces. Consequently, no convex regular-hedron can be obtained by composing the square pyramid and one of the 12 solids listed above.

Enlarging the pyramid $M_{2}$ by the solid $M_{8}$ along the square face of $M_{2}$, we get a nonconvex polyhedron. This can be checked with the help of the algebraic model of $M_{8}$; see 6, 7]. Finally, the sphenocorona $M_{22}$ (Figure 34) has a single superfundamental face. Adjoining the pyramid to it, we get an $F$-polyhedron, because each of its faces contains a vertex incident to five edges. This $F$-polyhedron is $\mathrm{P}_{2,26}=\mathrm{M}_{2}+\mathrm{M}_{22}=\mathrm{J}_{87}$.

Proposition 5 describes all composite solids obtained by composing pyramids $M_{3}$ and rotundas $M_{9}$. Therefore, when considering the corresponding solids, we shall use that proposition and its proof. In particular, two pyramids $M_{3}$ can be composed to obtain only one convex regular-hedron, namely, $\mathrm{P}_{2,27}=\mathrm{M}_{3}+\mathrm{M}_{3}=\mathrm{J}_{13}$.

Each Zalgaller or Ivanov solid that stands in Table 2 after $M_{3}$ has at most one fundamental pentagonal face. Compositions along it lead to the polyhedra $P_{2,28}, \ldots, P_{2,32}$, placed in Table 3, and to the $F$-polyhedra

$$
P_{2,33}=\overline{M_{3}+M_{20}}, \quad P_{2,34}=\overline{M_{3}+Q_{4}} .
$$

The composition of the Pryakhin solid $Q_{6}$ with the pyramid $M_{3}$ along their superfundamental pentagons is the polyhedron $\overline{M_{3}+Q_{6}}=P_{2,33}$ treated before.

Joining two cupolas $M_{4}$, we can get only two convex regular-hedra: $P_{2,35}$ and $P_{2,36}$. Their projections can be seen in Figures 57 and 58 respectively, where the triangular orthobicupola $J_{27}$ and the cuboctahedron $[3,4,3,4]$ are enlarged to the top left and to the bottom right by the pyramids $M_{2}$. Each polyhedron that stands below $M_{4}$ in Table 2 admits composition with the cupola $M_{4}$ only along the hexagonal face. Among these polyhedra, only $M_{10}, M_{20}$, and $Q_{6}$ have such faces; composing them with $M_{4}$ yields the 
convex regular-hedra

$$
\mathrm{P}_{2,37}=\mathrm{M}_{4}+\mathrm{M}_{10}=\mathrm{J}_{65} \text { and } \mathrm{P}_{2,38}=\overline{\mathrm{M}_{4}+\mathrm{M}_{10}^{\prime}} .
$$

The regular-hedron $P_{2,38}$ will be called the augmented truncated tetrahedron.

Compositions of the square cupolas $M_{5}$ with itself and with the polyhedra that stand below it in Table 2 lead to the $F$-polyhedra

$$
\mathrm{P}_{2,39}=\mathrm{M}_{5}+\mathrm{M}_{5}=\mathrm{J}_{28}, \quad \mathrm{P}_{2,40}=\mathrm{M}_{5}+\mathrm{M}_{5}^{\prime}=\mathrm{J}_{29}
$$

and the convex regular-hedra

$$
P_{2,41}=M_{5}+M_{11}=J_{66} \quad \text { (Figure 551) }, \quad P_{2,42}=\overline{M_{5}+M_{11}^{\prime}} \text { (Figure 566), }
$$

the second of which will be called the augmented truncated cube.

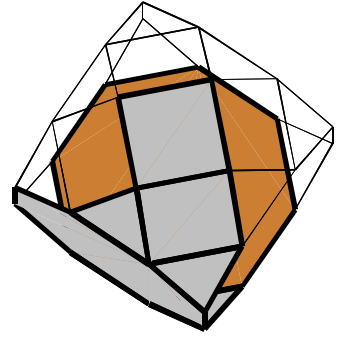

Figure 55. $P_{2,41}$.

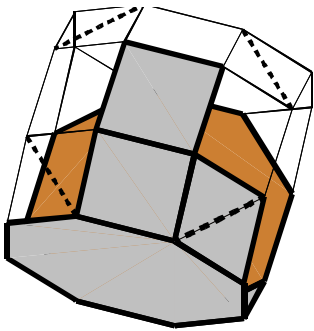

Figure 56. $P_{2,42}$.

The pentagonal cupola has one superfundamental face. Some compositions along this decagon were analyzed in Proposition [6. Now it suffices to add compositions with the rotunda $M_{9}$ and with the truncated rhombicosidodecahedra $M_{13}, M_{14}$. In this way we find the $F$-polyhedra

$$
\mathrm{P}_{2,43}=\mathrm{M}_{6}+\mathrm{M}_{6}=\mathrm{J}_{31}, \quad \mathrm{P}_{2,44}=\mathrm{M}_{6}+\mathrm{M}_{6}^{\prime}=\mathrm{J}_{30}
$$

and eight regular-hedra to include in Table 3 , namely, $P_{2, m}$ with $m=45,46, \ldots, 52$.

If we compose any of the polyhedra $M_{7}, M_{8}, \ldots, M_{15}, M_{20}, M_{22}, Q_{4}, Q_{6}$ with itself or with a polyhedron that stands below it in Table 2, we get no convex regular-hedra except for the solids $P_{2,53}$ and $P_{2,54}$ treated in Proposition 5

3.2. 3-composite polyhedra. The process of constructing the 3-composite polyhedra differs from that of 2-composite ones in the following way: instead of composing the polyhedra in Table 2 with polyhedra in the same table, now we must consider compositions of the polyhedra in Table 3 with those in Table 2. The 3-composite polyhedra created in this way and possessing a superfundamental face are placed in Table 4. Below we list all 3 -composite $F$-polyhedra:

$$
\begin{aligned}
\mathrm{P}_{3,2}=\overline{\Pi_{3}+\Pi_{4}+\Pi_{3}}, & \mathrm{P}_{3,3}=\overline{\Pi_{3}+\Pi_{4}+M_{2}}, \quad \mathrm{P}_{3,4}=\overline{\Pi_{3}+\Pi_{5}+M_{2},} \\
\mathrm{P}_{3,5}=\Pi_{3}+M_{1}+M_{1}=J_{14}, & \mathrm{P}_{3,7}=\Pi_{4}+M_{2}+M_{2}=J_{15}, \\
\mathrm{P}_{3,8}=\Pi_{5}+M_{2}+M_{2}=J_{53}, & \mathrm{P}_{3,9}=\Pi_{5}+M_{3}+M_{3}=J_{16}, \\
\mathrm{P}_{3,11}=\Pi_{6}+M_{2}+M_{2}^{\prime}=J_{56}, & \mathrm{P}_{3,12}=\Pi_{6}+M_{4}+M_{4}=J_{35}, \\
\mathrm{P}_{3,13}=\Pi_{6}+M_{4}+M_{4}^{\prime}=J_{36}, & \mathrm{P}_{3,14}=\Pi_{8}+M_{5}+M_{5}=[3,4,4,4], \\
\mathrm{P}_{3,15}=\Pi_{8}+M_{5}+M_{5}^{\prime}=J_{37}, & \mathrm{P}_{3,16}=\Pi_{10}+M_{6}+M_{6}=J_{38}, \\
\mathrm{P}_{3,17}=\Pi_{10}+M_{6}+M_{6}^{\prime}=J_{39}, & P_{3,23}=A_{4}+M_{2}+M_{2}=J_{17},
\end{aligned}
$$




$$
\begin{array}{ll}
P_{3,24}=A_{5}+M_{3}+M_{3}=[3,3,3,3,3], & P_{3,25}=A_{6}+M_{4}+M_{4}=J_{44}, \\
P_{3,26}=A_{8}+M_{5}+M_{5}=J_{45}, & P_{3,27}=A_{10}+M_{6}+M_{6}=J_{46}, \\
P_{3,34}=\overline{M_{3}+M_{8}+M_{3}}, & P_{3,40}=\overline{M_{3}+M_{9}^{\prime}+M_{6}}, \\
P_{3,41}=\overline{M_{3}+M_{9}^{\prime}+M_{6}^{\prime}}, & P_{3,45}=M_{5}+M_{11}+M_{5}=J_{67}, \\
P_{3,46}=\overline{M_{5}+M_{11}+M_{5}^{\prime}}, & P_{3,47}=\overline{M_{5}+M_{11}^{\prime}+M_{5}}, \\
P_{3,50}=M_{6}+M_{12}+M_{6}^{\prime \prime}=J_{69}, & P_{3,51}=\overline{M_{6}+M_{12}+M_{6}^{\prime \prime \prime}}, \\
P_{3,53}=\overline{M_{6}+M_{12}^{\prime}+M_{6}^{\prime}}(\text { Fig. [49)}, & P_{3,56}=M_{6}+M_{14}+M_{6}=[3,4,5,4], \\
P_{3,57}=M_{6}+M_{14}+M_{6}^{\prime}=J_{72}, & P_{3,58}=M_{6}+M_{14}^{\prime}+M_{6}=J_{73} .
\end{array}
$$

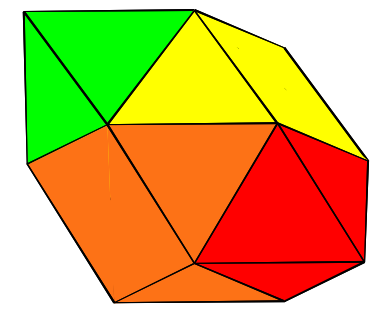

FiguRe 57. $P_{4,12}$.

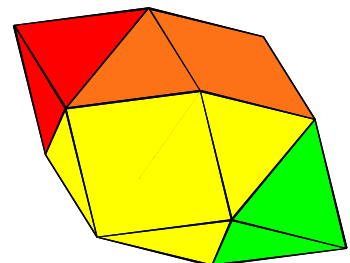

Figure 58. $P_{4,13}$.

3.3. 4-composite polyhedra. Composing each polyhedron occurring in Table 4 with the noncomposite polyhedra of Table 2, we arrive at a list of all 4-composite solids; see below and Table 5. The first two of them, $P_{4,1}=\overline{\Pi_{3}+\Pi_{3}^{\prime}+M_{2}+M_{2}}$ and $P_{4,2}=$ $\overline{\Pi_{3}+\Pi_{3}^{\prime}+\mathrm{M}_{2}+\mathrm{M}_{2}^{\prime}}$, will be called the rhombic prism augmented along adjacent faces and the rhombic prism augmented along parallel faces, respectively.

TABle 4. Polyhedra $P_{3, k}$ with nonempty sets $S F$

\begin{tabular}{|r|l|c|l|}
\hline$k$ & Name & Notation & $S F$ \\
\hline 1 & Augmented rhombic prism & $\Pi_{3}+\Pi_{3}^{\prime}+M_{2}$ & 4,4 \\
\hline 6 & $\begin{array}{l}\text { Bi-augmented triangular } \\
\text { prism }\end{array}$ & $\Pi_{3}+M_{2}+M_{2}, J_{50}$ & 4 \\
\hline 10 & $\begin{array}{l}\text { Parabi-augmented } \\
\text { hexagonal prism }\end{array}$ & $\Pi_{6}+M_{2}+M_{2}, J_{55}$ & 4 \\
\hline 18 & $\begin{array}{l}\text { Elongated pentagonal } \\
\text { orthocupolarotunda }\end{array}$ & $\Pi_{10}+M_{6}+M_{9}, J_{40}$ & 5 \\
\hline 19 & $\begin{array}{l}\text { Elongated pentagonal } \\
\text { gyrocupolarotunda }\end{array}$ & $\Pi_{10}+M_{6}+M_{9}^{\prime}, J_{41}$ & 5 \\
\hline 20 & $\begin{array}{l}\text { Elongated augmented } \\
\text { pentagonal rotunda }\end{array}$ & $\Pi_{10}+M_{9}+M_{3}$ & 10 \\
\hline 21 & $\begin{array}{l}\text { Elongated pentagonal } \\
\text { orthobirotunda }\end{array}$ & $\Pi_{10}+M_{9}+M_{9}, J_{42}$ & 5,5 \\
\hline 22 & $\begin{array}{l}\text { Elongated pentagonal } \\
\text { gyrobirotunda }\end{array}$ & $\Pi_{10}+M_{9}+M_{9}^{\prime}, J_{43}$ & 5,5 \\
\hline 28 & $\begin{array}{l}\text { Gyroelongated pentagonal } \\
\text { cupolarotunda }\end{array}$ & $A_{10}+M_{6}+M_{9}, J_{47}$ & 5 \\
\hline
\end{tabular}




\begin{tabular}{|c|c|c|c|}
\hline$k$ & Name & Notation & $S F$ \\
\hline 29 & $\begin{array}{l}\text { Gyrate augmented } \\
\text { elongated pentagonal rotunda }\end{array}$ & $A_{10}+M_{9}+M_{3}$ & 10 \\
\hline 30 & $\begin{array}{l}\text { Gyroelongated pentagonal } \\
\text { birotunda }\end{array}$ & $A_{10}+M_{9}+M_{9}, J_{48}$ & 5,5 \\
\hline 31 & Augmented octahedron & $M_{1}+M_{2}+M_{2}$ & 3 \\
\hline 32 & $\begin{array}{l}\text { Augmented triangular } \\
\text { orthobicupola }\end{array}$ & $M_{2}+M_{4}+M_{4}$ & 4 \\
\hline 33 & Augmented cuboctahedron & $M_{2}+M_{4}+M_{4}^{\prime}$ & 4 \\
\hline 35 & $\begin{array}{l}\text { Bi-augmented pentagonal } \\
\text { rotunda }\end{array}$ & $M_{3}+M_{9}+M_{3}$ & 10 \\
\hline 36 & $\begin{array}{l}\text { Pentagonal meta-augmented } \\
\text { gyrocupolarotunda }\end{array}$ & $M_{3}+M_{9}+M_{6}$ & 5 \\
\hline 37 & $\begin{array}{l}\text { Pentagonal meta-augmented } \\
\text { orthocupolarotunda }\end{array}$ & $M_{3}+M_{9}+M_{6}^{\prime}$ & 5 \\
\hline 38 & Augmented icosidodecahedron & $M_{3}+M_{9}+M_{9}$ & 5,5 \\
\hline 39 & $\begin{array}{l}\text { Meta-augmented pentagonal } \\
\text { orthobirotunda }\end{array}$ & $M_{3}+M_{9}+M_{9}^{\prime}$ & $5,5,5,5$ \\
\hline 42 & $\begin{array}{l}\text { Augmented pentagonal } \\
\text { orthobirotunda }\end{array}$ & $M_{3}+M_{9}^{\prime}+M_{9}$ & 5,5 \\
\hline 43 & Metabi-augmented dodecahedron & $M_{3}+M_{15}+M_{3}, J_{60}$ & 5 \\
\hline 48 & $\begin{array}{l}\text { Metabi-augmented truncated } \\
\text { dodecahedron }\end{array}$ & $M_{6}+M_{12}+M_{6}, J_{70}$ & 10 \\
\hline 49 & $\begin{array}{l}\text { Gyrate bimeta-augmented } \\
\text { truncated dodecahedron }\end{array}$ & $M_{6}+M_{12}+M_{6}^{\prime}$ & 10 \\
\hline 52 & $\begin{array}{l}\text { Bigyrate meta-augmented } \\
\text { truncated dodecahedron }\end{array}$ & $M_{6}+M_{12}^{\prime}+M_{6}$ & 10 \\
\hline 54 & $\begin{array}{l}\text { Metagyrate diminished } \\
\text { rhombicosidodecahedron }\end{array}$ & $M_{6}+M_{13}+M_{6}, J_{78}$ & 10 \\
\hline 55 & $\begin{array}{l}\text { Bigyrate diminished } \\
\text { rhombicosidodecahedron }\end{array}$ & $M_{6}+M_{13}^{\prime}+M_{6}, J_{79}$ & 10 \\
\hline
\end{tabular}

$$
\begin{aligned}
\mathrm{P}_{4,3} & =\Pi_{3}+\mathrm{M}_{2}+\mathrm{M}_{2}+\mathrm{M}_{2}=\mathrm{J}_{51}, & & \mathrm{P}_{4,4}=\Pi_{6}+\mathrm{M}_{2}+\mathrm{M}_{2}+\mathrm{M}_{2}=J_{57}, \\
\mathrm{P}_{4,5} & =\overline{\Pi_{10}+\mathrm{M}_{6}+\mathrm{M}_{9}+\mathrm{M}_{3}}, & \mathrm{P}_{4,6} & =\overline{\Pi_{10}+\mathrm{M}_{6}+\mathrm{M}_{9}^{\prime}+\mathrm{M}_{3}}, \\
\mathrm{P}_{4,9} & =\overline{\mathrm{A}_{10}+\mathrm{M}_{6}+\mathrm{M}_{9}+\mathrm{M}_{3}}, & \mathrm{P}_{4,11} & =\overline{M_{1}+\mathrm{M}_{2}+\mathrm{M}_{2}+\mathrm{M}_{1}}, \\
\mathrm{P}_{4,12} & =\overline{M_{2}+\mathrm{M}_{4}+\mathrm{M}_{4}+\mathrm{M}_{2}}, & \mathrm{P}_{4,13} & =\overline{M_{2}+\mathrm{M}_{4}+\mathrm{M}_{4}^{\prime}+\mathrm{M}_{2} .}
\end{aligned}
$$

The polyhedra $P_{4,12}$ and $P_{4,13}$ will be called the bi-augmented triangular orthobicupola (Figure 57) and the bi-augmented cubic octahedron (Figure 58), respectively.

$$
\begin{array}{ll}
P_{4,14}=\overline{M_{3}+M_{9}+M_{3}+M_{6}}, & P_{4,15}=\overline{M_{3}+M_{9}+M_{3}+M_{6}^{\prime}}, \\
P_{4,18}=\overline{M_{3}+M_{9}+M_{9}+M_{3}}, & P_{4,22}=\overline{M_{3}+M_{9}^{\prime}+M_{9}+M_{3}}, \\
P_{4,23}=M_{3}+M_{15}+M_{3}+M_{3}=J_{61}, & P_{4,24}=M_{6}+M_{12}+M_{6}+M_{6}=J_{71}, \\
P_{4,25}=\overline{M_{6}+M_{12}+M_{6}+M_{6}^{\prime}}, & P_{4,26}=\overline{M_{6}+M_{12}+M_{6}^{\prime}+M_{6}}, \\
P_{4,27}=\overline{M_{6}+M_{12}^{\prime}+M_{6}+M_{6}}, & P_{4,28}=M_{6}+M_{13}+M_{6}+M_{6}=J_{74}, \\
P_{4,29}=M_{6}+M_{13}^{\prime}+M_{6}+M_{6}=J_{75} . &
\end{array}
$$


3.4. 5-composite polyhedra. Each polyhedron occurring in Table 5 can be enlarged up to a convex regular-hedron only by adjoining the pyramid $M_{3}$; the last five of them were considered in Proposition 5. As a result, we obtain the following list of all 5composite $F$-polyhedra:

$$
\begin{array}{ll}
P_{5,1}=\overline{\Pi_{10}+M_{9}+M_{3}+M_{9}+M_{3}}, & P_{5,2}=\overline{\Pi_{10}+M_{9}+M_{3}+M_{9}^{\prime}+M_{3}}, \\
P_{5,3}=\overline{A_{10}+M_{9}+M_{3}+M_{9}+M_{3}}, & P_{5,4}=\overline{M_{3}+M_{9}+M_{3}+M_{9}+M_{3}}, \\
P_{5,7}=\overline{M_{3}+M_{9}+M_{3}+M_{9}^{\prime}+M_{3}^{\prime \prime} .} &
\end{array}
$$

TABLE 5. Polyhedra $P_{4, k}$ with nonempty sets $S F$

\begin{tabular}{|r|l|c|l|}
\hline$k$ & Name & Notation & $S F$ \\
\hline 7 & $\begin{array}{l}\text { Elongated augmented } \\
\text { pentagonal orthobirotunda }\end{array}$ & $\Pi_{10}+M_{9}+M_{3}+M_{9}$ & 5 \\
\hline 8 & $\begin{array}{l}\text { Elongated augmented } \\
\text { pentagonal gyrobirotunda }\end{array}$ & $\Pi_{10}+M_{9}+M_{3}+M_{9}^{\prime}$ & 5 \\
\hline 10 & $\begin{array}{l}\text { Augmented gyrate elongated } \\
\text { pentagonal birotunda }\end{array}$ & $A_{10}+M_{9}+M_{3}+M_{9}$ & 5 \\
\hline 16 & $\begin{array}{l}\text { Bimeta-augmented } \\
\text { icosidodecahedron }\end{array}$ & $M_{3}+M_{9}+M_{3}+M_{9}$ & 5 \\
\hline 17 & $\begin{array}{l}\text { Bimeta-augmented } \\
\text { birotunda }\end{array}$ & $M_{3}+M_{9}+M_{3}+M_{9}^{\prime}$ & $5,5,5$ \\
\hline 19 & $\begin{array}{l}\text { Bimeta-augmented birotunda } \\
\text { with adjacent rhombus }\end{array}$ & $M_{3}+M_{9}+M_{9}^{\prime}+M_{3}$ & 5,5 \\
\hline 20 & $\begin{array}{l}\text { Bi-augmented birotunda } \\
\text { without adjacent rhombus }\end{array}$ & $M_{3}+M_{9}+M_{9}^{\prime}+M_{3}^{\prime}$ & 5 \\
\hline 21 & $\begin{array}{l}\text { Augmented and meta-augmented } \\
\text { birotunda }\end{array}$ & $M_{3}+M_{9}+M_{9}^{\prime}+M_{3}^{\prime \prime}$ & 5 \\
\hline
\end{tabular}

TABle 6 . Polyhedra $P_{5, k}$ with nonempty sets $S F$

\begin{tabular}{|c|l|c|c|}
\hline$k$ & Name & Notation & $S F$ \\
\hline 5 & $\begin{array}{l}\text { Tri-augmented birotunda with } \\
\text { two pairs of adjacent rhombi }\end{array}$ & $M_{3}+M_{9}+M_{3}+M_{9}^{\prime}+M_{3}$ & 5,5 \\
\hline 6 & $\begin{array}{l}\text { Tri-augmented birotunda with } \\
\text { one pair of adjacent rhombi }\end{array}$ & $M_{3}+M_{9}+M_{3}+M_{9}^{\prime}+M_{3}^{\prime}$ & 5 \\
\hline
\end{tabular}

3.5. A convex 48-hedron composed of six noncomposite solids. The two regularhedra of Table 6 were found in Proposition 5. Consequently, the only convex regularhedron composed of six noncomposite solids is the 4-augmented pentagonal orthobirotunda (Figure 45)

$$
P_{6,1}=\overline{M_{3}+M_{9}+M_{3}+M_{9}^{\prime}+M_{3}+M_{3}} .
$$

Since $P_{6,1}$ is an $F$-polyhedron, there is no polyhedron $P_{7,1}$. This proves Theorem 1 . 


\section{§4. CONCLUSION}

In addition to 75 Johnson solids, four Archimedean solids, the icosahedron $P_{3,24}$, and the octahedron $P_{2,24}$, Theorem 1 indicates 68 convex regular-hedra, each with a conditional edge. Adding to these 92 Johnson solids, five Ivanov solids, and one Pryakhin solid, we see that the following is true.

Theorem 2. Besides the regular and the equiangular-semiregular polyhedra, there are exactly 166 convex regular-hedra.

\section{ACKNOWLEDGMENTS}

The author is grateful to V. A. Zalgaller and A. M. Gurin for fruitful discussions, and to the participants of algebraic and geometrical seminars and conferences in Limanchic, St.Petersburg branch of Steklov Mathematical Institute of RAS, Moscow State University, Moscow Center for Continuous Mathematical Education, Institute of Mathematics and Mechanics of the Ural Branch of Russian Academy of Sciences, and in Krasnoyarsk Pedagogical University for valuable remarks and suggestions.

\section{REFERENCES}

[1] A. M. Gurin and V. A. Zalgaller, To the history of the study of convex polyhedra with regular faces and faces composed of regular, Trudy S.-Peterburg. Mat. Obshch. 14 (2008), 215-294. (Russian)

[2] V. A. Zalgaller, Convex polyhedra with regular faces, Zap. Nauchn. Sem. Leningrad. Otdel. Mat. Inst. Steklov. (LOMI) 2 (1967), 220 pp. (Russian) MR0227860 (37:3444)

[3] B. A. Ivanov, Polyhedra with faces that are composed of regular polygons, Ukrain. Geom. Sb. No. 10 (1971), 20-34. (Russian) MR0301634 (46:790)

[4] Yu. A. Pryakhin, Convex polyhedra with regular faces, Ukrain. Geom. Sb. No. 14 (1973), 83-88. (Russian) MR0338930 (49:3693)

[5] A. V. Timofeenko, On classification of convex polyhedra with regular faces, Algebra and Logic (Internat. Russian-China Sem.), Irkut. Gos. Ped. Univ., Irkutsk, 2007, pp. 103-108. (Russian)

[6] - The non-Platonic and non-Archimedean noncomposite polyhedra, Fundam. i Prikl. Mat. 14 (2008), no. 2, 179-205; English transl., J. Math. Sci. 162 (2009), no. 5, 710-729. MR2475600

[7] _ Convex regular polyhedra that are not cut by any plane into regular polyhedral parts, Mat. Tr. (Novosibirsk, Akad. Nauk) 11 (2008), no. 1, 132-152. (Russian) MR2437485

[8] - On a junction of noncomposite polyhedra, Proc. of the Internat. School-Sem. on Geometry and Analysis in Memory of N. V. Efimov, Rostov-on-Don, 2008, pp. 70-72. (Russian)

[9] A. V. Timofeenko and A. M. Gurin, On the theory of convex polyhedra with regular faces, Dokl. Akad. Nauk 419 (2008), no. 3, 320-323. (Russian) MR2462091

[10] _ Algebraic and computer models of convex polyhedra with regular faces, and faces formed from regular polygons, Geometry in Odessa - 2008 (Internat. Conf., thesis) (V. V. Gol'dberg, V. M. Kuzakon', A. G. Kushner, and V. V. Lychagin, eds.), Odessa, 2008, pp. 131-132. (Russian)

[11] N. W. Johnson, Convex polyhedra with regular faces, Canad. J. Math. 18 (1966), 169-200. MR0185507 (32:2973)

[12] V. A. Zalgaller, Convex polyhedra with regular faces, Sem. in Math. Steklov Math. Inst., Leningrad, vol. 2, Consultants Bureau, New York, 1969. MR0240719 (39:2064)

Institute of Computational Modeling, Siberian Branch, Russian Academy of Sciences, AcAdemgorodoK 50/44, Krasnoyarsk 660036, Russia

E-mail address: A.V.Timofeenko62@mail.ru

Received 31/AUG/2008

Translated by A. PLOTKIN 